\title{
Building an explicit de Sitter
}

\author{
Jan Louis, ${ }^{a, b}$ Markus Rummel, ${ }^{a}$ Roberto Valandro ${ }^{a}$ and Alexander Westphal ${ }^{c}$ \\ ${ }^{a}$ II. Institut für Theoretische Physik der Universität Hamburg, \\ D-22761 Hamburg, Germany \\ ${ }^{b}$ Zentrum für Mathematische Physik, Universität Hamburg, \\ D-22761 Hamburg, Germany \\ ${ }^{c}$ Theory Group, Deutsches Elektronen-Synchrotron DESY, \\ D-22603 Hamburg, Germany \\ E-mail: jan.louis@desy.de, markus.rummel@desy.de, \\ roberto.valandro@desy.de, alexander.westphal@desy.de
}

ABSTRACT: We construct an explicit example of a de Sitter vacuum in type IIB string theory that realizes the proposal of Kähler uplifting. As the large volume limit in this method depends on the rank of the largest condensing gauge group we carry out a scan of gauge group ranks over the Kreuzer-Skarke set of toric Calabi-Yau threefolds. We find large numbers of models with the largest gauge group factor easily exceeding a rank of one hundred. We construct a global model with Kähler uplifting on a two-parameter model on $\mathbb{C P}_{11169}^{4}$, by an explicit analysis from both the type IIB and F-theory point of view. The explicitness of the construction lies in the realization of a D7 brane configuration, gauge flux and RR and NS flux choices, such that all known consistency conditions are met and the geometric moduli are stabilized in a metastable de Sitter vacuum with spontaneous GUT scale supersymmetry breaking driven by an F-term of the Kähler moduli.

KEYwords: Flux compactifications, F-Theory, dS vacua in string theory, Superstring Vacua

ARXiv EPRINT: 1208.3208 


\section{Contents}

1 Introduction \& motivation 2

2 Constraints on large gauge group rank in the landscape $\quad 6$

2.1 D7-branes from the IIB perspective $\quad 7$

2.2 D7-branes from the F-theory perspective 9

2.3 Maximal gauge group ranks 12

3 The type IIB perspective of $\mathbb{C P}_{11169}^{4}[18] \quad 13$

$\begin{array}{lll}3.1 & \text { Geometric set-up and orientifold involution } & 14\end{array}$

$\begin{array}{lll}3.2 & \text { D7-brane configuration } & 17\end{array}$

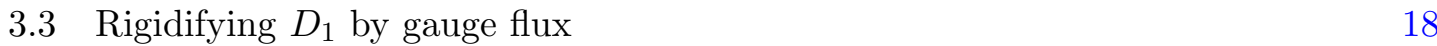

3.4 Avoiding D-terms and zero-modes from matter fields 20

3.5 D3 tadpole cancellation condition 22

4 The F-theory perspective of $\mathbb{C P}_{11169}^{4}[18]$

5 Moduli stabilization in the large volume limit 25

$6 \quad$ A fully stabilized de Sitter vacuum of $\mathbb{C P}_{11169}^{4}[18] \quad 27$

$\begin{array}{lll}6.1 & \text { Kähler uplifted de Sitter vacua } & 27\end{array}$

$\begin{array}{lll}6.2 & \text { Complex structure moduli } & 30\end{array}$

7 Conclusions $\quad 34$

$\begin{array}{ll}\text { A Models from two line weight systems } & 36\end{array}$

A.1 One hypersurface Calabi-Yaus 36

$\begin{array}{lll}\text { A.2 Complete intersections } & 37\end{array}$

B The $\operatorname{Sp}(N)$ resolved fourfold $\quad 41$

B.1 Geometry of the $\operatorname{Sp}(N)$ resolved fourfold 41

B.2 Intersections of exceptional divisors from the Stanley-Reisner ideal 42

B.3 Arithmetic genus of divisors 44

C Geometry of the SU(N) resolved fourfold 46 


\section{Introduction \& motivation}

String theory is a candidate for a fundamental theory of nature since it has the capacity to describe chiral matter fermions with non-Abelian gauge interactions within a consistent theory of quantum gravity. However, at a more detailed level it turns out to be difficult to clearly identify the Standard Model in an expanding universe as one of the possible backgrounds. Part of the problem arises from the poor conceptual understanding of string theory which still is largely based on perturbative formulations. As a consequence there is an abundance of perturbatively consistent backgrounds - each with a generically highdimensional moduli space.

This moduli space is particularly apparent if one views the string backgrounds geometrically, that is as compactifications of a ten-dimensional (10D) space-time on some compact six-dimensional manifold. Imposing $4 \mathrm{D} \mathcal{N}=1$ space-time supersymmetry for phenomenological reasons singles out a specific class of six-manifold which include CalabiYau (CY) manifolds. These manifolds have a large number of non-trivial deformations (moduli) associated with their volume and shape. In a low energy effective description the moduli correspond to 4D massless scalar fields which are flat directions of the scalar potential. Their stabilization has been a long-standing problem but in recent years significant progress has been made at least for certain classes of compactifications. ${ }^{1}$

The mechanism relevant for this paper uses quantized vacuum expectation values (VEVs) for $p$-form gauge field strengths of type IIB string theory [5, 6]. These fluxes generate a scalar potential for a large fraction of the typically $\mathcal{O}(100)$ moduli and potentially stabilizes them in a local minimum. The remaining moduli are then fixed by a combination of non-perturbative effects (such as gaugino condensation of $4 \mathrm{D} \mathcal{N}=1$ gauge theories living on D-branes) [7], a combination of perturbative and non-perturbative effects [8], or an interplay of perturbative effects and negative curvature of the internal space alone [9-16].

The mechanism for moduli stabilization can simultaneously break supersymmetry (SUSY) spontaneously by generating non-vanishing $F$ - and/or $D$-terms [17, 18]. Alternatively, SUSY breaking can be achieved by inserting an additional quasi-explicit source, such as an anti-brane in a warped region [7]. The vacuum energy of such fully stabilized compactifications with SUSY breaking can be both positive and negative, leading to a description of de Sitter (dS) space as metastable vacua of compactified string theory $[7,8,17-26]$. The number of these $\mathrm{dS}$ vacua is exponentially large due to large number of topologically distinct fluxes necessary for moduli stabilization in the first place. In type IIB string theory compactified on a warped 6D Calabi-Yau manifold there are typically $\mathcal{O}(100)$ complex structure or shape moduli associated to the three-dimensional topologically non-trivial subspaces (three-cycles) of the Calabi-Yau. On each three-cycle a flux can be turned on and thus one has $\mathcal{O}(100)$ fluxes to choose for stabilizing all the complex structure moduli. For, say, 10 available flux quanta per three-cycle this yields $\mathcal{O}\left(10^{100}\right)$ isolated potential dS vacua [27-29]. This exponentially large number of dS vacua with a flat number density distribution of the vacuum energy is often called the 'landscape'

\footnotetext{
${ }^{1}$ For recent reviews of flux compactifications and their associated uplifts to dS, and a much more comprehensive bibliography, see e.g. [1-4].
} 
of string vacua. It is coupled to the populating processes of Coleman-deLuccia tunneling and eternal inflation, which together realize space-time regions filled with each $\mathrm{dS}$ vacuum infinitely often. As a consequence Weinberg's anthropic argument for the smallness of the present-day cosmological constant can be realized in string theory. Hence the landscape of dS vacua gave string theory the ability to accommodate recent data from observational cosmology which demonstrated late-time accelerated expansion of our visible Universe, consistent with an extremely small positive cosmological constant $\Lambda \sim 10^{-122} M_{\mathrm{P}}^{4}{ }^{2}$

For the purpose of this work we will restrict ourselves to type IIB warped Calabi-Yau orientifold compactifications with three-form flux which arise as a specific weak coupling limit (Sen limit) of F-theory on elliptically fibered CY fourfolds [35, 36]. This will allow us to use the known techniques for constructing CYs, calculate their topological data and derive the $4 \mathrm{D}$ effective theory. Supersymmetry breaking and lifting the AdS vacuum to dS typically is the least reliable step. Therefore, our goal is to construct explicit global models in the above type IIB context, which exhibit the dynamics of Kähler uplifting. ${ }^{3}$ In such models the interplay of gaugino condensation on D7-branes and the leading $\mathcal{O}\left(\alpha^{\prime 3}\right)$ correction of the Kähler potential fix the Kähler moduli in a SUSY breaking minimum, after three-form flux has supersymmetrically stabilized the complex structure moduli and the axio-dilaton. The vacuum energy of this minimum can be dialed from AdS to dS by adjusting the fluxes appearing in the superpotential. Both SUSY breaking and lifting to dS are driven by an F-term of the Kähler moduli sector which is induced by the presence of the $\alpha^{\prime}$ correction $[17,24,38]$. The dS uplift is therefore spontaneous and arises from the geometric closed string moduli. This is the motivation for trying to construct a fully explicit consistent global model including a choice for the flux. Such a model yields an example for a 4D dS space in string theory which is explicit within the limit of the currently available knowledge.

In this work we discuss constructions of Kähler uplifted dS vacua on compact CalabiYau manifolds. We perform this analysis both from perspective of F-theory and its weak coupling limit of type IIB string theory compactified on warped Calabi-Yau orientifolds. F-theory arises from the observation that the type IIB axio-dilaton can vary over the compactification manifold $B_{3}$. One then interprets the axio-dilaton as the complex structure modulus of an elliptic curve fibered over the threefold $B_{3}$, realizing a complex fourdimensional manifold that takes the form of an elliptically fibered Calabi-Yau fourfold in order to ensure $\mathcal{N}=1$ supersymmetry in the $4 \mathrm{D}$ effective theory. The attraction of F-theory is due to the geometrization of the complete non-perturbative super Yang-Mills (SYM) dynamics of stacks of 7-branes wrapping four-cycles in type IIB in terms of resolvable ADE type singularities in the fourfold. Of particular interest here are F-theory compactifications on elliptically fibered fourfolds which admit a global weak coupling limit (Sen limit), where the axio-dilaton goes to weak string coupling and becomes approxi-

\footnotetext{
${ }^{2}$ The flatness of the vacuum energy distribution on the landscape has recently been questioned by studies using random matrix techniques in general [30-32], and a statistical analysis of combined input parameter distributions in the context of Kähler uplifting [33, 34].

${ }^{3}$ In this work, we are not addressing the question of constructing a standard model like sector in combination with moduli stabilization, as it has been recently achieved in [26, 37]. Here, we simply want to avert these complicating features for the sake of moduli stabilization in a stable de Sitter vacuum.
} 
mately constant everywhere on the threefold base of the fibration. In this limit, the set of 7-branes located at the locus where the fiber degenerates, can be described entirely in terms of perturbative O7-planes and D7-branes.

The analysis in [24] looked at the effective dynamics of moduli stabilization via Kähler uplifting. A class of possible examples consists of a Calabi-Yau threefold with an expression for the volume $\mathcal{V}$ given by the sum of one large four-cycle modulus and a collection of smaller so called blow-up four-cycle moduli. This resembles the structure of a swiss cheese with its overall volume given mainly by just the size of the enclosing cycle and many tiny holes (the blow-up four-cycles). We know in addition that in Kähler uplifted dS vacua the volume $\mathcal{V}$ of the type IIB Calabi-Yau scales with the rank $N$ of the condensing gauge group as $\mathcal{V} \propto N^{3 / 2}$. Moreover, the scale of Kähler moduli stabilization and thus the resulting Kähler moduli masses are suppressed by an additional $\mathcal{O}(1 / \mathcal{V})$ compared to the scale of the flux-induced complex structure moduli stabilization. These features lead us to search for condensing gauge groups with a large rank which induce a large volume.

We will consider models which can be easily uplifted to F-theory compactified on elliptically fibered CY fourfolds that are hypersurfaces in an ambient toric variety [39-41]. In addition, we insist on the existence of a smooth Sen limit, as we use the leading $\alpha^{\prime}$ correction which is not understood in F-theory in general. The type IIB Calabi-Yau threefold is then the double cover of the F-theory base manifold $B_{3}$. It is described by an equation such as $\xi^{2}=h$, where the orientifold involution is realized by $\xi \mapsto-\xi$. Here $\xi$ denotes one of the holomorphic complex projective coordinates of the ambient toric variety where the CY threefold lives, with the orientifold plane sitting at $\xi=0$. The data describing the D7-brane stacks and the orientifold planes in F-theory via ADE singularities can be specified in terms of sections of holomorphic line bundles and the corresponding homology classes of the associated divisors. The D7-brane tadpole forces the D7-brane to wrap cycles whose homology classes (denoted by $[D 7]$ ) sum up to $[D 7]=8[O 7]=8[\{\xi=0\}]$ in the type IIB CY threefold. To obtain a large gauge group one generically has to wrap many branes on certain divisors. This is only possible if the coefficients of the orientifold class $[O 7]=\sum_{i} c_{i}\left[D_{i}\right]$ are large (here the $D_{i}$ denote a complete set of divisor four-cycles which in turn form a base of the 2nd Dolbeault cohomology group $H^{1,1}$ ). Increasing the homology class [O7] introduces singularities in the base manifold which have to be resolved. Moreover, the Calabi-Yau threefold hypersurface in the weak coupling limit should be free of singularities as well, and therefore the orientifold planes should not intersect each other. This in general turns out to be a severe constraint when one tries to increase the class of the orientifold by choosing the weights defining the toric variety appropriately.

Finally, we have to check that all used divisors are rigid such that gaugino condensation does contribute to the superpotential. In this context, the role of gauge flux is crucial: On the one hand, a suitable choice can 'rigidify' a divisor by fixing some of its deformation moduli $[42,43]$ (see [44-46] for discussions in the F-theory context). On the other hand, switching on gauge flux can generate additional zero modes in the form of chiral matter (especially at the intersection of branes) which forbids the contribution of gaugino condensation in the superpotential. Moreover, the presence of fluxes can be required by the necessity of canceling the Freed-Witten anomaly [47, 48]. 
As mentioned before, the dynamics of Kähler uplifting was demonstrated so far on 'swiss cheese' type Calabi-Yau threefolds. The cheese with its one big bounding cycle and its many tiny holes implies a certain form of the volume. Let us assume a CY with a set of divisor four-cycles $D_{i}$ whose (real) volumes we denote by $\mathcal{V}_{i}$. Then the volume of the a swiss cheese $\mathrm{CY}$ is defined by $\mathcal{V} \sim \mathcal{V}_{1}^{3 / 2}-\sum_{i} \mathcal{V}_{i}^{3 / 2}$ or as $\mathcal{V} \sim\left(\mathcal{V}_{1}+\sum_{i} \mathcal{V}_{i}\right)^{3 / 2}-\sum_{i} \mathcal{V}_{i}^{3 / 2}$ for an approximately swiss cheese $\mathrm{CY}$. In this situation one can manufacture a large overall volume by enforcing a large gauge group rank on the corresponding divisor $D_{1}$ which in turn leads to a large $\mathcal{V}_{1}$. A potential complication may then arise as a high number of branes on $D_{1}$ typically enforces singularities on other divisors which might yield the overall volume small even though $\mathcal{V}_{1}$ is large.

At the end, we combine the Kähler moduli stabilization with an explicit dilaton and complex structure moduli stabilization via RR and NS fluxes. We discuss a specific hypersurface in the weighted projective space $\mathbb{C P}_{11169}^{4}$ as a concrete example where the whole program can be executed. In this case, discrete symmetries of the complex structure moduli space and a specific choice of the three-form fluxes allow us to fix all the complex structure explicitly along the lines of [49]. We check that this choice of flux results in values for $W_{0}$ which are such that the Kähler stabilization leads to a metastable dS vacuum. Hence, our model constitutes an example for a dS space in string theory which is explicit within the limits of existing knowledge. The only implicitness that remains is the unknown complex structure moduli dependence of the 1-loop determinant prefactor of the non-perturbative effect. Recent work has shown [24] that for large volume the mass scale of the Kähler moduli separates from the scale of the axio-dilaton and the complex structure moduli by one inverse power of the volume. This justifies replacing the complex structure moduli by their VEVs inside the 1-loop determinants, and allows us to parametrize these prefactors as effective constants. Moreover, we can clearly dial the VEVs of the complex structure moduli by availing ourselves of the exponentially large flux discretuum, which easily accounts for a potential mild tuning of the value of the 1-loop determinants.

This paper is organized as follows. In section 2 we study the constraints for having a gauge group with large rank by discussing Kreuzer-Skarke models [50] and hypersurfaces in toric varieties. For the subclass of threefolds with an elliptic F-theory lift $\left(\sim 10^{5}\right.$ models) we scan and extract the distribution of the largest-rank gauge group as a function of the number of Kähler moduli $h^{1,1}$. Then we choose to consider $\mathbb{C P}_{11169}^{4}[18]$ as an explicit example and construct large-rank ADE gauge groups on a choice of two divisors, and analyze the consistency constraints both in the type IIB weak-coupling limit, and from the F-theory perspective in sections 3 and 4, respectively. Section 5 reviews the general results for supersymmetric flux stabilization of the complex structure moduli and the axio-dilaton. In section 6 we study the scalar potential that stabilizes the Kähler moduli. We single out a band in the $g_{s}-W_{0}$ plane where one finds de Sitter vacua. Here $W_{0}$ denotes the VEV of the superpotential which arises from supersymmetric flux stabilization of the complex structure moduli. For the explicit model on $\mathbb{C P}_{11169}^{4}[18]$, we show how to fix explicitely all the complex structure moduli, thanks to a particular symmetry of the moduli space and a special choice of three-form fluxes. Finally, we check that this choice of flux results in values for $W_{0}$ which are such that the Kähler stabilization leads to a metastable dS vacuum. We 
conclude and discuss our results in section 7. More details of the toric resolution of the $\mathrm{Sp}(k)$-singularity can be found in the appendices. We have kept the steps of our calculations rather explicit for future reference but also since certain aspects of the arguments are often only implicit in the existent literature.

\section{Constraints on large gauge group rank in the landscape}

In this section, we discuss generic constraints on obtaining large gauge group gaugino condensation which is a crucial input for the method of Kähler uplifting. In the context of non-compact Calabi-Yaus, it was already discussed in [51] that arbitrarily high gauge group ranks are possible. As we will see, the situation in the compact case is more restrictive. We will mostly discuss the perturbative type IIB picture and conclude with some remarks about non-perturbative F-theory models at the end of this section.

Our laboratory will be the landscape of complex three-dimensional Calabi-Yau manifolds that are hypersurfaces in toric varieties. These were classified in [50] by constructing all 473,800,776 reflexive polyhedra that exist in four dimensions, yielding 30,108 distinct Hodge numbers of the corresponding Calabi-Yau manifolds $X_{3}$. For simplicity we will study a subset of these, i.e. the set of 184,026 maximal polytopes yielding 10,237 distinct Hodge numbers. These can be represented by a weight system of positive integers $n_{1}, \ldots, n_{5}$. To each integer $n_{i}$ one can associate one of the projective coordinates $\left\{u_{1}, \ldots, u_{4}, \xi\right\}$ of a four-dimensional toric space:

$$
\frac{u_{1} u_{2} u_{3} u_{4} \quad \xi}{n_{1} n_{2} n_{3} n_{4} n_{5}} \quad \text { with } \quad 0<n_{1} \leq n_{2} \leq n_{3} \leq n_{4} \leq n_{5}
$$

The integers $n_{i}$ determine the scaling equivalence relation the coordinates satisfy:

$$
\left(u_{1}, \ldots, u_{4}, \xi\right) \sim\left(\lambda^{n_{1}} u_{1}, \ldots, \lambda^{n_{4}} u_{4}, \lambda^{n_{5}} \xi\right), \quad \text { with } \lambda \in \mathbb{C}^{*} .
$$

The divisors $D_{i}:\left\{u_{i}=0\right\}$ and $D_{\xi}:\{\xi=0\}$ are called toric divisors. A hypersurface in such toric space is a Calabi-Yau (i.e. its first Chern class vanishes) if the degree of the defining equation is equal to $\sum_{i}^{5} n_{i}$.

Eq. (2.2) defines the complex four-dimensional projective space $\mathbb{C P}_{n_{1} n_{2} n_{3} n_{4} n_{5}}^{4}$. Often it is useful to think about the weights as defining a gauged linear sigma model (GLSM) [52]. If one of the weights $n_{i}$ is greater than one, the ambient space is not smooth. This is the case for any toric Calabi-Yau that is not the quintic, which is given by $n_{i}=1, \sum_{i}^{5} n_{i}=$ 5. The corresponding singularities have to be resolved if they intersect the Calabi-Yau hypersurface. The resolution process yields additional weights, i.e. eq. (2.1) becomes a $k \times(k+5)$ matrix, called the weight matrix, that defines the resolved toric ambient space $X_{4}^{\mathrm{amb}}$. Generically, the greater the $n_{i}$ in eq. (2.1), the more lines of weights have to be added to obtain a smooth Calabi-Yau. Often there is more than one choice to resolve the singularities, corresponding to different triangulations of the corresponding polytope. The number of lines of the weight matrix $k$ gives the dimension of $H^{1,1}\left(X_{4}^{\text {amb }}, \mathbb{Z}\right)$. Since some divisors of $X_{4}^{\mathrm{amb}}$ might either intersect $X_{3}$ in two or more disconnected and independent 
divisors of $X_{3}$, or even not intersect $X_{3}$ all, $\operatorname{dim} H^{1,1}\left(X_{4}^{\text {amb }}, \mathbb{Z}\right)$ is not necessarily the same as $h^{1,1}=\operatorname{dim} H^{1,1}\left(X_{3}, \mathbb{Z}\right)$. However, increasing $k$ will generically also increases $h^{1,1}$.

To realize an $\mathcal{N}=1$ supersymmetric compactification of type IIB in four dimensions and to consistently include D-branes and fluxes we introduce $\mathrm{O} 7$ orientifold planes in the construction. For simplicity, we only consider orientifold projections $\mathcal{O}=(-1)^{F} \Omega_{p} \sigma$ acting via the holomorphic involution

$$
\sigma: \quad \xi \mapsto-\xi,
$$

i.e. the sign of the coordinate with the highest weight is reversed. We demand

$$
n_{\xi} \equiv n_{5}=\sum_{i=1}^{4} n_{i},
$$

such that the Calabi-Yau hypersurface equation symmetric under (2.3) is given by

$$
\xi^{2}=P_{\left(2 \sum_{i}^{4} n_{i}, \ldots\right)} .
$$

The dots denote possible additional weights that have to be added to obtain a threefold free of singularities. Note that eq. (2.5) only holds if $n_{\xi}=\sum n_{i}$ also for the resolution weights which we assume is in many cases possible and which we have verified in various examples.

Hence, all information of the Calabi-Yau threefold is stored in the weights $n_{1}, \ldots, n_{4}$ and the chosen triangulation. Moreover, the resolution of the three dimensional manifold $\mathbb{C P}_{n_{1} n_{2} n_{3} n_{4}}^{3}$ is the base $B_{3}$ of the elliptically fibered fourfold that realizes the uplift of the type IIB model to F-theory. For this reason, models fulfilling eq. (2.4), are named models of the 'F-theory type'. These are 97,036 weight systems leading to 7,602 distinct pairs of Hodge numbers. The first Chern class of $B_{3}$ defines a non-trivial line bundle, the anticanonical bundle $\bar{K}$, with $\bar{K}=c_{1}\left(B_{3}\right)$ (in this paper we use the same symbol to denote the line bundle and its corresponding divisor class). Due to eq. (2.4) the homology class of the O7-plane at $\xi=0$ is given as $[O 7]=\bar{K}$.

\subsection{D7-branes from the IIB perspective}

Now, we discuss the inclusion of D7-branes from the IIB perspective. The presence of the O7-plane induces a negative D7 charge of $-8[O 7]$. This has to be compensated by the positive charge of the D7-brane stacks $[D 7]$ ([D7] is the homology class of the surface wrapped by the D7-brane configuration). In other words, since $[O 7]=\bar{K},[D 7]$ has to be given by the vanishing locus of a section of $\bar{K}^{8}$ to saturate the D7 tadpole. More specifically, it was found in [53] that for a single invariant D7-brane saturating the D7 tadpole cancellation condition, its world volume is given by the (non-generic) polynomial equation

$$
\eta^{2}-\xi^{2} \chi=0
$$

with $\eta$ and $\chi$ sections of $\bar{K}^{4}$ and $\bar{K}^{6}$, respectively. (For practical purposes, $\eta$ and $\chi$ can be seen as polynomials in the complex coordinates $u_{i}$ of the resolved base manifold $B_{3}$.) This brane can be understood as the result of the recombination of one standard D7-brane wrapping the surface $\eta-\xi \psi=0$ with its orientifold image, wrapping $\eta+\xi \psi=0$. In fact, 
such a brane configuration is described by the vanishing locus of the factorized polynomial $(\eta-\xi \psi)(\eta+\xi \psi)=\eta^{2}-\xi^{2} \psi^{2}$. By adding to this polynomial the term $\xi^{2}\left(\psi^{2}-\chi\right)$, i.e. by recombining the two factors, one obtains the equation (2.6). The resulting recombined invariant D7-brane is called in literature 'Whitney brane', as it has the singular shape of the so called Whitney umbrella [53].

For non-generic forms of the polynomials $\eta$ an $\chi$, the Whitney brane can split into different stacks. In particular a stack of $2 N_{i}$ branes wrapping the invariant toric divisor $D_{i}:\left\{u_{i}=0\right\}$ manifests itself via the factorization

$$
\eta=u_{i}^{N_{i}} \tilde{\eta}, \quad \chi=u_{i}^{2 N_{i}} \tilde{\chi},
$$

such that eq. (2.6) becomes

$$
u_{i}^{2 N_{i}}\left(\tilde{\eta}^{2}-\xi^{2} \tilde{\chi}\right)=0
$$

where on the invariant divisor at $u_{i}=0$ there is an $\operatorname{Sp}\left(N_{i}\right)$ stack and $\tilde{\eta}^{2}-\xi^{2} \tilde{\chi}$ describes a Whitney brane of lower degree. Since the Whitney brane has always to be described by a holomorphic equation, $N_{i}$ cannot be made arbitrarily large.

For $u_{i=1, \ldots, 4}$ we can be more specific. Eq. (2.8) becomes

$$
u_{i}^{2 N_{i}}\left(\tilde{\eta}_{\left(4 n_{\xi}-n_{i} N_{i}, \ldots\right)}^{2}-\xi^{2} \tilde{\chi}_{\left(6 n_{\xi}-2 n_{i} N_{i}, \ldots\right)}\right)=0
$$

where the dots denote the degrees that are imposed via the weight system of the resolved ambient space $X_{4}^{\mathrm{amb}}$. If the degree in the first scaling is the most restrictive we obtain the strongest bound from the holomorphicity of $\tilde{\chi}$, i.e.

$$
N_{i} \leq 3 \frac{n_{\xi}}{n_{i}} .
$$

Due to the ordering of the $n_{i}$, eq. (2.1), we expect to be able to put the largest number of branes on the divisor $D_{1}$ and the constraining quantity is the largest integer $N_{\mathrm{lg}}$ that is smaller than $3 n_{\xi} / n_{1}$. $N_{\text {lg }}$ will serve as our large gauge group indicator in the following.

Before we proceed, let us make a few comments on the choice of the large gauge group indicator that were in part already addressed in the introduction:

- For $n_{1}=1$, the first column of the $k \times(k+5)$ weight matrix describing the resolved ambient space $X_{4}^{\mathrm{amb}}$ is always given by $(1,0, \ldots, 0)^{T}$ and hence $N_{\lg }$ is always the limiting quantity. However, for $n_{1}>1$ the first column of the weight matrix has to contain additional non-zero entries smaller than $n_{1}$ to resolve the singularities. The holomorphicity of the Whitney brane equation in the corresponding degree could in principle be more restraining than $N_{\mathrm{lg}}$. Even if this would be the case we still expect $N_{\lg }$ to give a right estimate since in the $\mathcal{O}(10)$ examples where we have computed the resolved weight matrix, using PALP [54-56], it was always the most restrictive.

- The type of gauge group enforced by the $2 N_{1}$ branes depends on the geometry of the O7-plane and the gauge flux. If the divisor is invariant under $\xi \mapsto-\xi$ and transverse to the O7-plane, we have an $\operatorname{Sp}\left(N_{1}\right)$ gauge group that can be broken by gauge flux to $\mathrm{SU}\left(N_{1}\right)$. The Coxeter numbers are $N_{1}+1$ for $\operatorname{Sp}\left(N_{1}\right)$ and $N_{1}$ for $\operatorname{SU}\left(N_{1}\right)$. If the divisor lies on the orientifold plane the gauge group is $\mathrm{SO}\left(2 N_{1}\right)$ with Coxeter number $2 N_{1}-2$. 
- To check if the brane-stack contributes to the superpotential in a suitable way for the method of Kähler uplifting one has to fulfill additional constraints. First of all, in order to have a pure SYM theory that undergoes gaugino condensation, possible light matter fields must be forbidden. A sufficient condition is that the wrapped divisor is rigid and that the brane intersections and world volume should not produce additional chiral zero-modes. Note that with growing rank of the weight matrix, $D_{1}$ tends to be rigid since it typically cannot be deformed into other toric divisors. Furthermore, the volume form of the threefold has to be of the approximately swiss cheese type. Finally, the factorization in eq. (2.9) should not force a further factorization of the remaining Whitney brane in toric divisors which enter the volume form with a negative sign. In fact, since gaugino condensation forces the volumes of these divisors to be large, this would make the overall volume small. This does not necessarily have to be a problem since in the approximately swiss cheese type the enforced brane stacks on other toric divisors might also increase the overall volume.

We mention these points to make it clear that the indicator $N_{\mathrm{lg}}$ only serves as an easily computable estimate for the largest gauge group rank one can obtain in a threefold of the F-theory type. To see if one can stabilize the Kähler moduli in a large volume, one has to check the additional constraints case by case. We will do this in section 3, constructing a consistent model of a Kähler uplifted de Sitter vacuum.

\subsection{D7-branes from the F-theory perspective}

Let us now discuss the constraints on the large gauge group rank in the perturbative limit of F-theory. This theory is physically equivalent to weakly coupled type IIB, discussed in the previous section. However, the geometric F-theory picture provides a different perspective and a cross check of our results.

Before we discuss the D7-brane setup in F-theory let us set the stage. To obtain an $\mathcal{N}=1$ effective four dimensional effective theory starting from 12-dimensional F-theory we have to compactify on an elliptically fibered Calabi-Yau fourfold. More specifically, the fourfold can be described as a hypersurface in an ambient fivefold which is a $\mathbb{C P}_{123}^{2}$ fibration over a three dimensional base $B_{3}$, i.e. one introduces three additional complex coordinates coordinates and a scaling relation

$$
(X, Y, Z) \sim\left(\lambda^{2} X, \lambda^{3} Y, \lambda Z\right) .
$$

As far as the scaling in the classes of the base is concerned $Z$ scales as a section of the canonical bundle $K$ of $B_{3}$, in order to ensure the Calabi-Yau condition of the fourfold. The elliptically fibered Calabi-Yau fourfold can be defined by the Weierstrass model

$$
Y^{2}=X^{3}+f X Z^{4}+g Z^{6},
$$

with $f$ and $g$ being sections of $\bar{K}^{4}$ and $\bar{K}^{6}$, respectively. However, for the purpose of detecting singularities it is more convenient to bring (2.12) in the Tate form $[57,58]$ :

$$
Y^{2}+a_{1} X Y Z+a_{3} Y Z^{3}=X^{3}+a_{2} X^{2} Z^{2}+a_{4} X Z^{4}+a_{6} Z^{6}
$$




\begin{tabular}{|c|cccccc|}
\hline & $a_{1}$ & $a_{2}$ & $a_{3}$ & $a_{4}$ & $a_{6}$ & $\Delta$ \\
\hline $\mathrm{Sp}(N)$ & 0 & 0 & $N$ & $N$ & $2 N$ & $2 N$ \\
$\mathrm{SU}(2 N)$ & 0 & 1 & $N$ & $N$ & $2 N$ & $2 N$ \\
$\mathrm{SU}(2 N+1)$ & 0 & 1 & $N$ & $N+1$ & $2 N+1$ & $2 N+1$ \\
$\mathrm{SO}(4 N+1)$ & 1 & 1 & $N$ & $N+1$ & $2 N$ & $2 N+3$ \\
$\mathrm{SO}(4 N+2)$ & 1 & 1 & $N$ & $N+1$ & $2 N+1$ & $2 N+3$ \\
$\mathrm{SO}(4 N+3)$ & 1 & 1 & $N+1$ & $N+1$ & $2 N+1$ & $2 N+4$ \\
$\mathrm{SO}(4 N+4)$ & 1 & 1 & $N+1$ & $N+1$ & $2 N+1$ & $2 N+4$ \\
\hline
\end{tabular}

Table 1. The exponent $w_{i}$ in (2.14) is given for all $a_{i}$ and $\Delta$ and for different singularities. The discriminant $\Delta$ depends on the $a_{i}$ according to eq. (2.21).

where the Tate polynomials $a_{i}$ are functions of the base coordinates $u_{i}$ such that they are sections of $\bar{K}^{i}$. The Tate form (2.13) and the Weierstrass form (2.12) of the defining equation can be related by completing the square and the cube and shifting the $X, Y$ coordinates.

In F-theory, D7-branes manifest themselves via singularities of the elliptic fibration. To engineer a singularity on a divisor $D_{j}:\left\{u_{j}=0\right\}$ the Tate polynomials have to factorize as

$$
a_{i}=u_{j}^{w_{i}} a_{i, w_{i}}
$$

with positive integer numbers $w_{i}$ encoding which kind of singularity is realized. Since $a_{i, w_{i}}$ has to be holomorphic, $w_{i}$ cannot be made arbitrarily large. For a tabular overview of the possible resolvable singularities that can arise in such a construction see [59]. The singularities with Coxeter number larger than 30 are either of the Sp, SU or SO type (see table 1).

We can again analyze the constraints on the maximal gauge group rank in more detail. Since the anti-canonical class of the base $B_{3}$ is given by $\bar{K}=\sum_{i=1}^{4} D_{i}$ and $n_{\xi}=\sum_{i=1}^{4} n_{i}$, eq. (2.14) can be written as

$$
a_{\left(i n_{\xi}, \ldots\right)}=u_{j}^{w_{i}} a_{\left(i n_{\xi}-w_{i} n_{j}, \ldots\right)}
$$

where the dots once more denote scalings originating from the resolution of the singularities of the original weight system of the base. If one considers the singularities listed in table 1 , the most severe constraints regarding holomorphicity of eq. (2.15) come from $a_{3}$ and $a_{6}$. A sufficient condition for the $a_{i}$ to always be holomorphic at least under the first scaling is

$$
N_{j} \equiv w_{3}=\frac{w_{6}}{2} \leq 3 \frac{n_{\xi}}{n_{j}}
$$

which is exactly what we found in eq. (2.10) in the type IIB picture. Thus, also from the F-theory perspective we arrive at the large gauge group indicator $N_{\mathrm{lg}}$. The caveats discussed in the comments following eq. (2.10) of course also have to be taken into account in the F-theory picture.

So far our F-theory discussion has been for generic values of the string coupling. However, we eventually want to obtain a stable de Sitter vacuum by using the leading $\alpha^{\prime}$ correction to the Kähler potential [60] which is only known in perturbative type IIB. As 
long as this correction remains unknown in non-perturbative F-theory, we have to restrict our analysis to Sen's weak coupling limit $g_{s} \rightarrow 0$ [36].

In the Tate form (2.13), the Sen limit [36] is imposed by the rescalings [59]

$$
a_{3} \mapsto \epsilon a_{3}, \quad a_{4} \mapsto \epsilon a_{4}, \quad a_{6} \mapsto \epsilon^{2} a_{6}
$$

and $\epsilon \rightarrow 0$. The string coupling is related to the parameter $\epsilon$ by

$$
g_{s} \sim-\frac{1}{\log |\epsilon|} \rightarrow 0 \quad \text { as } \quad \epsilon \rightarrow 0 .
$$

Completing the square and the cube in eq. (2.13) gives a relation between $f, g$ in the Weierstrass model and the Tate polynomials:

$$
f=-\frac{1}{48}\left(h^{2}-24 \epsilon \eta\right), \quad g=-\frac{1}{864}\left(-h^{3}+36 \epsilon h \eta-216 \epsilon^{2} \chi\right),
$$

with

$$
h=a_{1}^{2}+4 a_{2}, \quad \eta=a_{1} a_{3}+2 a_{4}, \quad \chi=a_{3}^{2}+4 a_{6},
$$

where $h, \eta$ and $\chi$ are sections of $\bar{K}^{2}, \bar{K}^{4}$ and $\bar{K}^{6}$ respectively. The discriminant locus $\Delta=0$, where the elliptic fiber degenerates, gives the location of the D7/O7-planes. The discriminant is given by

$$
\Delta=\frac{1}{16}\left(\epsilon^{2} h^{2} P_{D 7}+8 \epsilon^{3} \eta^{3}+27 \epsilon^{4} \chi^{2}-9 h \epsilon^{3} \eta \chi\right) \sim \frac{1}{16} \epsilon^{2} h^{2} P_{D 7}+\mathcal{O}\left(\epsilon^{3}\right) .
$$

where $P_{D 7}=-\frac{1}{4}\left(\eta^{2}-h \chi\right)$. We see that in the weak coupling limit $\epsilon \rightarrow 0$, the locus $\Delta=0$ splits into two components. Studying the monodromies of the elliptic fiber around such components, one realizes that at $h=0$ there is an O7-plane, while at $P_{D 7}=0$ there is a D7-brane [36]. The type IIB Calabi-Yau hypersurface that is a double cover of $B_{3}$ is given by

$$
X_{3}: 0=\xi^{2}-h=\xi^{2}-\left(a_{1}^{2}+4 a_{2}\right) .
$$

Using this equation, we see that the relation defining the D7-brane configuration in the Calabi-Yau threefold is

$$
P_{D 7}=0, \quad \text { i.e. } \quad \eta^{2}-\xi^{2} \chi=0 \text {. }
$$

As said above, a brane wrapping such a surface has the form of a Whitney umbrella [53] and will be called a 'Whitney brane' in this paper.

Note that there is no restriction on $f$ and $g$ or the Tate polynomials $a_{i}$ respectively in the Sen limit. Hence, a singular configuration over a divisor $D_{j}$ enforced via a factorization $a_{i}=u_{j}^{w_{i}} a_{i, w_{i}}$ remains intact in the weak coupling limit. This is consistent with the fact that we found the same bound on the gauge group rank in the IIB picture and in the general F-theory picture.

However, one has to make sure that a non-singular Sen limit exists. For example, $\mathrm{SU}(N)$ singularities generically introduce conifold singularities in the type IIB Calabi-Yau threefold (located on top of the O7-plane) [59]. In case of $\mathrm{SU}(N)$ singularities, the set of 
F-theory bases that lead to a smooth type IIB Calabi-Yau threefold is only a subset of the singular free bases whose elliptic fibrations generate F-theory fourfolds [61]. Therefore, we expect the constraints on the gauge group rank to be less strict in a generic F-theory compactification. In F-theory the constraints are that the base is non-singular and that one is not forced to introduce fibre singularities that cannot be resolved according to the Kodaira or Tate classification. Hence, it would be very interesting if one could derive the analogue of the perturbative $\alpha^{\prime}$ correction to the Kähler potential in general F-theory, making the construction of truly non-perturbative de Sitter vacua accessible.

\subsection{Maximal gauge group ranks}

In this section, we give the results of our scan for the maximal gauge group indicator $N_{\mathrm{lg}}$ in the 97,036 models of the F-theory type contained in the classification of [50]. We use PALP to calculate the Hodge numbers resulting in 7,602 distinct pairs of Hodge numbers. We refer to this set of weight systems as the general set. We also gather all weight systems that lead to the same pair of Hodge numbers and choose as the representative the weight system with the smallest $N_{\mathrm{lg}}$ corresponding to the most conservative estimate for the maximal gauge group. This set of weight systems is referred to as the conservative set. We restrict our attention to manifolds with negative Euler number $\chi=2\left(h^{1,1}-h^{2,1}\right)$, further reducing the set of weight systems to 8,813 corresponding to 3,040 distinct pairs of $\left(h^{1,1}, h^{2,1}\right)$. We do this since $\chi<0$ is a necessary condition to apply the method of Kähler uplifting.

Our results are summarized in figure 1 and table 2 . The maximal $N_{\lg }$ we obtain is 2,330 in the general set and 806 in the conservative set. The minimal $N_{\lg }$ is 12 in both sets corresponding to the base $\mathbb{C P}_{1111}^{3}$. The mean $\bar{N}_{\text {lg }}$ we find is 204.5 in the general set and 132.8 in the conservative set. Generically, the critical value for Kähler uplifting to be in large volume regime $(\mathcal{V} \gtrsim 100)$ is $N_{\mathrm{lg}}^{\text {crit. }}=30$. Since the actual volume also depends on the intersection numbers and the stabilized volumes of the divisors other than $D_{1}, N_{\mathrm{lg}}^{\text {crit. }}=30$ can only serve as an estimate for large volume. The subset of weight systems with $N_{\mathrm{lg}}<$ $N_{\lg }^{\text {crit. }}$ is 444 in the general set and 267 in the conservative set, corresponding to only $5 \%$ respectively $9 \%$ of the models where the method of Kähler uplifting is not applicable.

Another important feature we notice is the dependence of $N_{\lg }$ on $h^{1,1}$. We see from figure 1 that $N_{\lg }$ tends to increase with $h^{1,1}$. In other words, if one wants to have very large gauge groups one has to buy this by a rather high number of Kähler moduli which of course has the disadvantage of increasing the complexity of the model, especially if it is not swiss cheese. ${ }^{4}$ The tendency of $N_{\mathrm{lg}} \propto h^{1,1}$ can be explained from the weight system: As $n_{\xi}=\sum_{i}^{4} n_{i}$ becomes large, a large number of lines has to be added to the weight matrix to make the threefold singularity free which generically increases the number of Kähler moduli.

We conclude this section with the remark that the possibility to engineer large enough gauge groups to obtain a large volume in the framework of Kähler uplifting is a generic feature of the landscape region we have analyzed.

\footnotetext{
${ }^{4}$ For an algorithm to check for the swiss cheese property of a threefold see [62].
} 


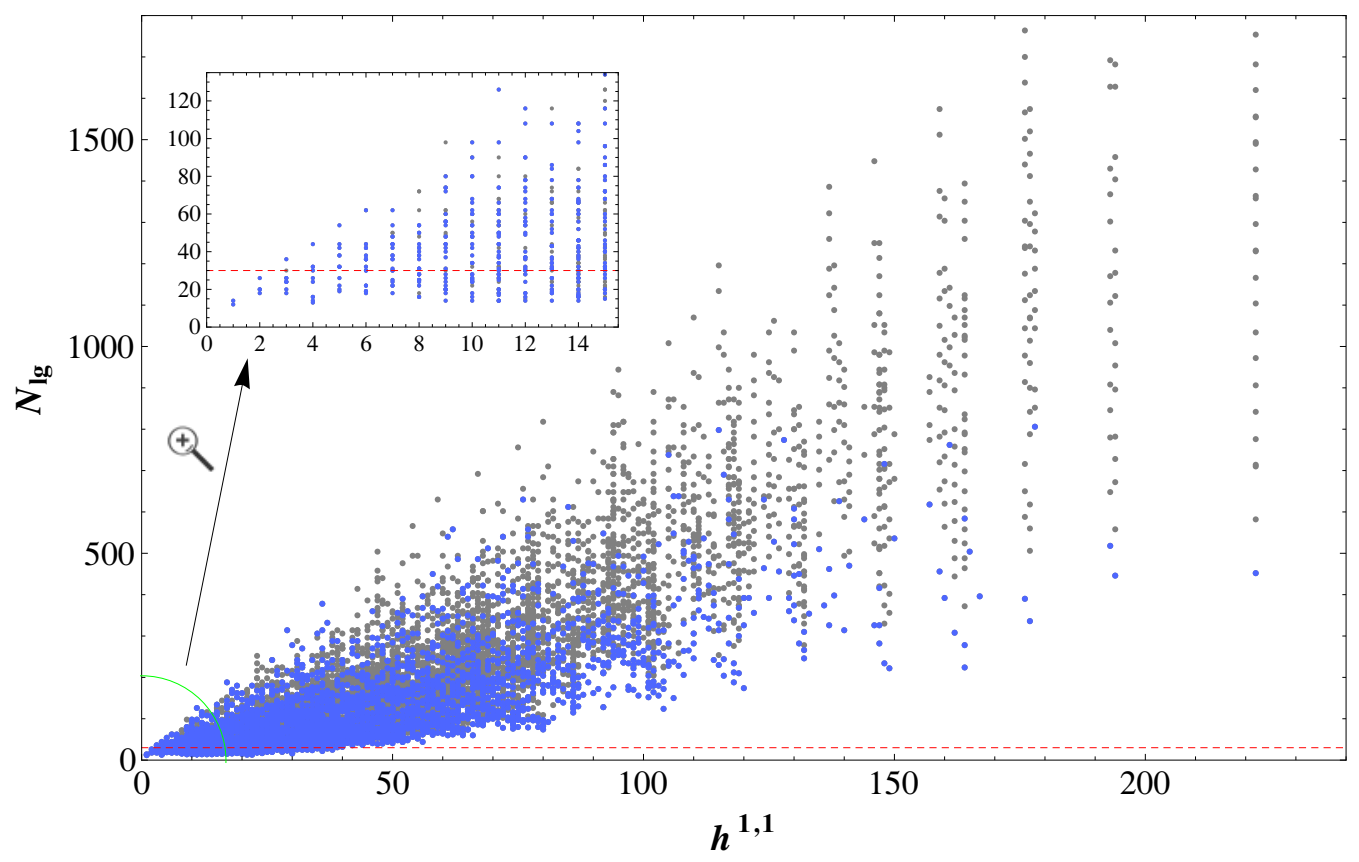

Figure 1. The large gauge group indicator $N_{\mathrm{lg}}$ as a function of $h^{1,1}$. The grey dots denote the general set of models, while the blue dots denote the conservative set (for explanations see text). The red dashed line denotes the critical gauge group rank for Kähler uplifting $N_{\lg }^{\text {crit. }}=30$.

\begin{tabular}{|l|ccccccc|c|}
\hline$N_{\mathrm{lg}}$ & $12-60$ & $61-210$ & $211-360$ & $361-510$ & $511-660$ & $661-806$ & $807-2330$ & $\sum$ \\
\hline$\#_{\text {gen. }}$ & 1964 & 4101 & 1435 & 592 & 313 & 162 & 246 & 8813 \\
$\#_{\text {cons. }}$ & 889 & 1590 & 409 & 115 & 30 & 7 & 0 & 3040 \\
\hline
\end{tabular}

Table 2. The distribution of $N_{\mathrm{lg}}$. The first line denotes the binning, while the second and third line contain the number of models in the respective $N_{\lg }$ intervals for the general and conservative set, respectively.

\section{The type IIB perspective of $\mathbb{C P}_{11169}^{4}[18]$}

In this section, we present an explicit example of a brane and gauge flux setup on a threefold of the landscape region studied in section 2. To keep the analysis tractable we study a threefold with small $h^{1,1}$. Looking at figure 1 , we see that there is a model with $h^{1,1}=2$ that has $N_{\mathrm{lg}}=27$, which is close to the critical value $N_{\mathrm{lg}}^{\text {crit. }}=30$. This is the CalabiYau threefold $X_{3}$ that is a degree 18 hypersurface in $\mathbb{C P}_{11169}^{4}$ (it is usually denoted as $\left.\mathbb{C P}_{1169}^{4}[18]\right)$. The corresponding weight system of the ambient toric space after resolving the singularities is

$$
X_{4}^{\mathrm{amb}}: \begin{array}{cccccc}
u_{1} & u_{2} & u_{3} & u_{4} & u_{5} & \xi \\
\hline 1 & 1 & 1 & 6 & 0 & 9 \\
0 & 0 & 0 & 1 & 1 & 2
\end{array}
$$

The two lines determine the two scaling equivalence relations that the coordinates satisfy (see eq. (2.2)). The Stanley-Reisner (SR) ideal encodes which homogeneous coordinates 
are not allowed to vanish simultaneously in the toric variety. For the four dimensional ambient space eq. (3.1) it is given by

$$
S R_{X_{4}^{\mathrm{amb}}}=\left\{u_{1} u_{2} u_{3}, u_{4} u_{5} \xi\right\}
$$

We will see in section 6 that on this manifold we can stabilize the two Kähler moduli to values corresponding to a volume $\mathcal{V} \simeq 52$. In constructing an explicit brane and gauge flux setup on $X_{3}$ we address the following issues that are crucial in constructing a global model [26, 37, 63-65]:

- The choice of the orientifold involution determines the class of the O7-plane. D7tadpole cancellation then implies $[D 7]=-8[O 7]$, fixing the degrees of the polynomial defining the D7-brane configuration. Requiring the presence of a D7-brane stack on $D_{1}$ with maximal gauge group rank $N_{\lg }$ might force the defining polynomial to factorize further, leading the presence of another large rank stack (section 3.2). Due to the swiss cheese structure of the volume form, this might destroy the large volume approximation and one has to check that this does not happens.

- To lift unwanted zero modes that might destroy gaugino condensation on some D7brane stacks, one needs to 'rigidify' the wrapped non-rigid toric divisors (in the present case $D_{1}$ ). To do this, the gauge flux has to be properly adjusted (section 3.3).

- To avoid the introduction of additional zero modes, due to non-zero gauge flux of the pull-back type (possibly forced by Freed-Witten anomaly cancellation [47, 48]), one has to choose such a flux in a proper way (section 3.4).

- One has to saturate the D3-tadpole cancellation condition (section 3.5).

In this specific model, we do not have to worry about the D5-tadpole cancellation. In fact, we will choose an orientifold involution with no odd $(1,1)$-forms, i.e. $h_{-}^{1,1}=0$. Hence, the D5-charge induced by gauge fluxes on D7-branes is automatically cancelled (there are no other source of D5-charge). In the present model, also K-theoretic torsion charges are cancelled $[66,67] .^{5}$

\subsection{Geometric set-up and orientifold involution}

Before we go through the points listed above one by one, let us mention some geometric properties of $X_{3}$ that will be needed during the following analysis. The Calabi-Yau is a hypersurface in the ambient space (3.1), defined by the equation

$$
\xi^{2}=P_{18,4}\left(u_{i}\right) \equiv u_{5} Q_{18,3},
$$

\footnotetext{
${ }^{5}$ According to the probe argument presented in [68], such charges are cancelled in a given set-up if any probe invariant D7-brane with gauge group $\mathrm{SU}(2)$ has an even number of zero modes in the fundamental representation. This is realized in our model, as we always have an even number of D7-branes in the chosen stacks.
} 
where $Q_{18,3} \equiv u_{5}^{3} P_{18}+u_{5}^{2} u_{4} P_{12}+u_{5} u_{4}^{2} P_{6}+u_{4}^{3}$. The factorization of the polynomial $P_{18,4}$ is enforced by its weights. The Hodge numbers of $X_{3}$ are $h^{1,1}=2$ and $h^{2,1}=272$. The (holomorphic) orientifold involution is given by

$$
\xi \mapsto-\xi
$$

This involution has $h_{-}^{1,1}=0$ and then the number of invariant Kähler moduli is $h_{+}^{1,1}=$ $h^{1,1}=2$ (as one can find a two dimensional basis of $H^{1,1}$ in which each divisor is invariant under the orientifold involution). The orientifold plane is located at the fixed point locus of the involution (3.4). In our case, the codimension three fixed locus is empty and hence we do not have O3-planes. On the other hand we have O7-planes on the codimension one fixed locus at $\xi=0$. Looking at equation (3.3), we see that this locus splits into two pieces. The corresponding four-cycles are given by the following equations in the ambient fourfold

$$
O 7_{u_{5}}:\left\{\xi=0 \cap u_{5}=0\right\}, \quad O 7_{Q}:\left\{\xi=0 \cap Q_{18,3}=0\right\} .
$$

These hypersurfaces in $X_{3}$ are not complete intersections of one equation with the defining CY equation (3.3), but are four-cycles of the ambient fourfold that intersect nontransversely the CY $X_{3}$ and then are four-cycles also in $X_{3}$. Using the SR-ideal, one sees that these two four-cycles do not intersect each other on the Calabi-Yau $X_{3}$. One can show that the divisor $O 7_{u_{5}}$ is a rigid divisor in the threefold $X_{3}$. Its homology class in the ambient space is $D_{\xi} \cdot D_{5}=\left[X_{3}\right] \cdot \frac{D_{5}}{2}$. We see that the class of this integral four-cycle in the threefold is $D_{5}^{\text {fix }}=\frac{D_{5}}{2}$. So we can use it as an element of an integral basis.

The threefold described so far is the double cover of the three dimensional base manifold

$$
B_{3}: \begin{array}{ccccc}
u_{1} & u_{2} & u_{3} & u_{4} & u_{5} \\
\hline 1 & 1 & 1 & 6 & 0 \\
0 & 0 & 0 & 1 & 1
\end{array} .
$$

The toric divisors of $B_{3}$, defined by the equations $u_{i}=0$ will be called $\hat{D}_{i}$, in order to distinguish them from their double covers $D_{i}$ in $X_{3}$ (given by the complete intersection $\left\{u_{i}=0\right\} \cap\left\{\xi^{2}-P_{18,4}=0\right\}$ in $\left.X_{4}^{\mathrm{amb}}\right)$.

The first Chern class of the base $B_{3}$ is $c_{1}\left(B_{3}\right)=\bar{K}=9\left[\hat{D}_{1}\right]+2\left[\hat{D}_{5}\right]$ and its SR ideal is given by

$$
S R_{B_{3}}=\left\{u_{1} u_{2} u_{3}, u_{4} u_{5}\right\} .
$$

Eq. (3.7) can be used to derive triple intersections of the base divisors [69, 70]

$$
\hat{D}_{1}^{3}=0, \quad \hat{D}_{1}^{2} \hat{D}_{5}=1, \quad \hat{D}_{1} \hat{D}_{5}^{2}=-6, \quad \hat{D}_{5}^{3}=36,
$$

where we have chosen the Poincaré duals of $\hat{D}_{1}$ and $\hat{D}_{5}$ as a basis of $H^{1,1}\left(B_{3}, \mathbb{Z}\right)$. If the divisors intersect away from the fixed point locus, the intersections in the double cover threefold $X_{3}$ are simply twice the intersections in the base. Before we have seen that their double covers $D_{1}, D_{5}$ do not form an integral basis of $H^{1,1}\left(X_{3}, \mathbb{Z}\right)$, i.e. there are integral divisors of $X_{3}$ that are rational combination of $D_{1}$ and $D_{5}$. Instead, an integral basis is given by $D_{1}, D_{5}^{\mathrm{fix}}$. Keeping in mind that $X_{3}$ is a double cover of $B_{3}$, that $D_{1}, D_{5}$ are the 
double covers of $\hat{D}_{1}, \hat{D}_{5}$ and that $D_{5}^{\text {fix }}=\frac{1}{2} D_{5}$, we can determine the intersection numbers on $X_{3}$ :

$$
D_{1}^{3}=0, \quad D_{1}^{2} D_{5}^{\mathrm{fix}}=1, \quad D_{1} D_{5}^{\mathrm{fix}^{2}}=-3, \quad D_{5}^{\mathrm{fix}^{3}}=9 .
$$

One can check these results by realizing that the threefold $X_{3}$ can also be described as a hypersurface in the toric ambient space

$$
X_{4}^{\mathrm{amb}, 123}: \quad \begin{array}{llllll}
u_{1} & u_{2} & u_{3} & x & y & z \\
\hline & 1 & 1 & 6 & 9 & 0 \\
0 & 0 & 0 & 2 & 3 & 1
\end{array}
$$

with Stanley-Reisner (SR) ideal

$$
S R_{X_{4}^{\mathrm{amb}, 123}}=\left\{u_{1} u_{2} u_{3}, x y z\right\} .
$$

We have used PALP to verify that the Calabi-Yau threefolds described by the respective hypersurfaces in the ambient toric varieties (3.1) and (3.10) are equivalent. At the level of their defining polytopes one sees this by finding that the normal forms [71] of the two polytopes are identical. ${ }^{6}$

The defining equation of $X_{3}$ as an hypersurface in $X_{4}^{\text {amb123 is }}$

$$
y^{2}=x^{3}+f_{12}\left(u_{1}, u_{2}, u_{3}\right) x z^{4}+g_{16}\left(u_{1}, u_{2}, u_{3}\right) z^{6} .
$$

The orientifold involution $\xi \mapsto-\xi$ is mapped to $y \mapsto-y$. The fixed locus in the ambient space is given by two components, i.e. $y=0$ and $z=0$ (the last one can be found once we apply the involution plus the second line equivalence relation in (3.10)). These loci intersect transversely the Calabi-Yau $X_{3}$ and the intersections are connected. So we again find that the fixed point set in $X_{3}$ is given by two disconnected components. We can identify $D_{5}^{\mathrm{fix}}=D_{z}$ and $D_{Q}^{\mathrm{fix}}=D_{y}$. Furthermore, the divisors $D_{i=1,2,3}$ are identified with the corresponding ones in $X_{4}^{\text {amb }}(3.1)$. By using $P A L P$, and making the given identifications, we again obtain the triple intersections (3.9). Moreover we obtain the second Chern class of the threefold:

$$
c_{2}(X)=102 D_{1}^{2}+69 D_{1} D_{5}^{\mathrm{fix}}+11 D_{5}^{\mathrm{fix}}{ }^{2} .
$$

Expanding the Kähler form of $X_{3}$ as $J=t_{1} D_{1}+t_{5} D_{5}^{\text {fix }}$, we find an approximately swiss cheese volume of the threefold

$$
\mathcal{V}=\frac{1}{6} \int_{X} J \wedge J \wedge J=\frac{1}{2}\left(t_{1}^{2} t_{5}-3 t_{1} t_{5}^{2}+3 t_{5}^{3}\right)=\sqrt{\frac{2}{3}}\left(\mathcal{V}_{1}+\frac{1}{3} \mathcal{V}_{5}\right)^{3 / 2}-\frac{\sqrt{2}}{9} \mathcal{V}_{5}^{3 / 2}
$$

with $\mathcal{V}_{i}=\partial \mathcal{V} / \partial t_{i}$. The Kähler cone is given by

$$
t_{1}-3 t_{5}>0, \quad t_{5}>0 .
$$

Finally, we list the hodge numbers, arithmetic genus $\chi_{0}$ and Euler number $\chi$ of divisors $D_{1}$ and $D_{5}^{\mathrm{fix}}$ in the threefold in table 3 . The results have been obtained by means of PALP and cohom Calg $[72,73] . D_{5}^{\mathrm{fix}}$ is rigid and hence fulfills the sufficient condition to contribute to the gaugino condensation superpotential. $D_{1}$ is not rigid, but one can choose a proper gauge flux on the wrapped D7-branes, that fixes the $h^{2,0}$ deformations. We will perform this calculation in section 3.3.

\footnotetext{
${ }^{6}$ We thank A.P. Braun for discussion on this point.
} 


\begin{tabular}{|c|cccccc|}
\hline & $h^{0,0}$ & $h^{1,0}$ & $h^{2,0}$ & $h^{1,1}$ & $\chi_{0}$ & $\chi$ \\
\hline$D_{1}$ & 1 & 0 & 2 & 30 & 3 & 36 \\
$D_{5}^{\text {fix }}$ & 1 & 0 & 0 & 1 & 1 & 3 \\
\hline
\end{tabular}

Table 3. Hodge numbers, arithmetic genus $\chi_{0}$ and Euler number $\chi$ of $D_{1}$ and $D_{5}$.

\subsection{D7-brane configuration}

Now, we discuss the inclusion of D7-branes, following the general procedure discussed in section 2.1. To cancel the D7-charge of the O7-planes at $\xi=0$, the equation describing the D7-brane configuration is given by (see eq. (2.6))

$$
\eta_{36,8}^{2}-\xi^{2} \chi_{54,12}=0
$$

where we degrees of the $\eta$ and $\chi$ polynomials are dictated by the degrees of $\xi$ and by the requirement that $[D 7]=-8[O 7]=-8 D_{\xi}$. To realize an $\operatorname{Sp}\left(N_{1}\right)$ gauge group on the invariant divisor $D_{1}$ with $N_{1}=N_{\mathrm{lg}}=27$ one takes

$$
\eta_{36,8}=u_{i}^{27} \tilde{\eta}_{9,8}, \quad \chi_{54,12}=u_{i}^{54} \tilde{\chi}_{0,12}
$$

Eq. (3.16) then becomes

$$
u_{1}^{54}\left(\tilde{\eta}_{9,8}^{2}-\xi^{2} \tilde{\chi}_{0,12}\right)=0 .
$$

Since $\tilde{\chi}_{0,12}=u_{5}^{12}$, the Whitney brane splits into a brane and image brane due to the factorization $\left(\tilde{\eta}_{9,8}-\xi u_{5}^{6}\right)\left(\tilde{\eta}_{9,8}+\xi u_{5}^{6}\right)$. The Whitney brane can only carry a flux that is 'trivial' on the Calabi-Yau threefold (i.e. its Poincaré dual non-trivial two-cycle on the D7brane world volume is homologically trivial on the Calabi-Yau threefold), contrary to its split branes that in general carries a flux necessary for Freed-Witten anomaly cancellation. However, a non-trivial flux would generate additional (chiral) zero modes. We avoid them by choosing a degree of the Whitney brane polynomial, such that it does not split into a brane and its image. Furthermore, for the calculation of D3 charge of the Whitney brane, see section 3.5, we sacrifice two more gauge group ranks on $D_{1}$, realizing an $\operatorname{Sp}(24)$ gauge group:

$$
u_{1}^{48}\left(\tilde{\eta}_{12,8}^{2}-\xi^{2} \tilde{\chi}_{6,12}\right)=0 .
$$

We note that the polynomial $\tilde{\eta}_{12,8}$ is forced to factorize as $\tilde{\eta}_{12,8}=u_{5}^{8} \tilde{\eta}_{12}+u_{5}^{7} u_{4} \tilde{\eta}_{6}+$ $u_{5}^{6} u_{4}^{2}=u_{5}^{6} \tilde{\eta}_{12,2}$, while the polynomial $\tilde{\chi}_{6,12}$ must have the form $\tilde{\chi}_{6,12}=u_{5}^{12} \tilde{\chi}_{6}+u_{5}^{11} u_{4} \tilde{\chi}_{0}$. In the following we will tune the parameter $\tilde{\chi}_{0}$ to zero. In this way the D7-brane configuration is described by the equation

$$
u_{1}^{48} u_{5}^{12}\left(\tilde{\eta}_{12,2}^{2}-\xi^{2} \tilde{\chi}_{6,0}\right)=0 .
$$

Hence, we see that requiring the factorization of the D7-brane equation in order to produce an $\operatorname{Sp}(24)$ stack enforces a further factorization, in this case of $u_{5}$. Since $D_{5}=2 D_{5}^{\mathrm{fix}}$ lies on the O7-plane, this generates an $\mathrm{SO}(24)$ gauge group. ${ }^{7}$

\footnotetext{
${ }^{7}$ If we do not take $\chi_{0}=0$, one has a factorization as $u_{1}^{48} u_{5}^{12}\left(\tilde{\eta}_{12,2}^{2}-Q_{18,3} \tilde{\chi}_{6,1}\right)=0$, where we used $\tilde{\chi}_{6,12}=u_{5}^{12} \tilde{\chi}_{6}+u_{5}^{11} u_{4}=u_{5}^{11} \tilde{\chi}_{6,1}$ and $\xi^{2}=u_{5} Q_{18,3}$. From this we would conclude that there is an $\operatorname{SO}(24)$ gauge group on $D_{5}^{\text {fix }}$. However, as we will see in the F-theory language, the actual gauge group is $\mathrm{SO}(23)$.
} 
The same conclusions on the factorization can be obtained using the description of $X_{3}$ as a hypersurface in $X_{4}^{\text {amb,123 }}(3.10)$. Remember that in that description the orientifold locus is given by $D_{y}+D_{z}$. To cancel the D7-tadpole, we need a D7-brane configuration wrapping the divisor class $8\left(D_{y}+D_{z}\right)=8\left(9 D_{1}+4 D_{z}\right)$, i.e. it is given by the equation $P_{72,32}=0$. A polynomial with these degrees must factorize as $P_{72,32}=z^{8} P_{72,24}$. Hence there are eight branes wrapping the orientifold-plane divisor $D_{z}$ (realizing a group $\mathrm{SO}(8)$ if one does not require further factorizations). The D7-tadpole generated by the O7-plane wrapping $D_{y}$ is cancelled by a Whitney brane, defined by the equation $\eta_{36,12}^{2}-y^{2} \chi_{54,18}=0$. As above, we require an $\operatorname{Sp}(24)$ stack on $u_{1}=0: \eta_{36,12}=u_{1}^{24} \eta_{12,12}$ and $\chi_{54,18}=u^{48} \chi_{6,18}$. The polynomial $\eta_{12,12}$ and $\chi_{6,18}$ must factorize as $\eta_{12,12}=z^{8} \hat{\eta}_{12,4}$ and $\chi_{6,18}=z^{16} \hat{\chi}_{6,2}$ (as above, we will take $\left.\hat{\chi}_{6,2}=z^{2} \hat{\chi}_{6,0}\right)$. We see that we have 16 more D7-branes that wrap the O7-plane divisor $D_{z}$, realizing an $\mathrm{SO}(16+8)=\mathrm{SO}(24)$ gauge group.

\subsection{Rigidifying $D_{1}$ by gauge flux}

In this section we construct explicitly a gauge flux on the $\operatorname{Sp}(24)$ stack wrapping $D_{1}$, that fixes all the deformation moduli of these branes. The equation describing $D_{1}$ is $u_{1}=0$, which can be deformed to $u_{1}+\zeta_{2} u_{2}+\zeta_{3} u_{3}=0$. We see that we have two deformation moduli, consistent with the fact that $h^{2,0}\left(D_{1}\right)=2$. We need to lift such zero modes in order to avoid destroying gaugino condensation on the D7-brane stack wrapping $D_{1}$.

The rigidifying flux will be taken such that it is not a pull-back of a $C Y_{3}$ two-form. In this way, it will not generate additional chiral matter and will not enter in the D-term constraints. On the other hand, since $h^{2,0}\left(D_{1}\right) \neq 0$, this flux will constrain the holomorphic embedding of the D-brane, by the F-term constraint $\mathcal{F}^{2,0}=0$. This type of gauge flux was introduced first in [43] to fix the deformation moduli of a non-rigid divisor wrapped by an E3-instanton. The use of such a flux to fix the deformation moduli of D7-branes such that they can support gaugino condensation was suggested in [26]. Here we make this flux explicit, proving that it indeed fixes the unwanted deformations and computing its D3-tadpole contribution.

To construct the flux, we follow the procedure described in [43]: We have to identify holomorphically embedded curves in the CY threefold that do not admit holomorphic deformation. In fact, the condition $\mathcal{F}^{2,0}=0$ means that the Poincaré dual two-cycle in $D_{1}$ remains holomorphic when the divisor is deformed in the threefold. If we cannot deform the curves, then some of the deformation moduli of $D_{1}$ are fixed.

We will work in the case in which the threefold is described by a hypersurface in the ambient space (3.10) with the defining equation (3.12). The rigid curves cannot be described by the intersection of one equation with (3.12) and $u_{1}=0$. In fact these will be always holomorphic as we deform the $D_{1}$ equation. To visualize these rigid holomorphic curves in an algebraic way, we have to parametrize $g_{16}$ appropriately, i.e. $g_{16}=\psi_{8}^{2}+u_{1} \tau_{15}$ where $\psi_{8}$ and $\tau_{15}$ are polynomials of degree 8 and 15 in the $u_{i}$ coordinates. ${ }^{8}$ In this way

\footnotetext{
${ }^{8}$ An analogous ansatz was done in [46] for an elliptically fibered Calabi-Yau fourfold, in order to find rigid four-cycles inside the fourfold.
} 
we can write the CY equation as

$$
\left(y-\psi_{8}\right)\left(y+\psi_{8}\right)=x\left(x^{2}+f_{12} z^{4}\right)+u_{1} \tau_{15} z^{6} .
$$

The rigid curve we are interested is the $\mathbb{P}^{1}$

$$
\mathcal{C}: \quad y=\psi_{8} \quad \cap \quad x=0 \quad \cap \quad u_{1}=0 .
$$

Through exact sequences (following [74]), one shows that its normal bundle in the threefold is $\mathcal{O}(-1) \oplus \mathcal{O}(-1)$ and then it has no holomorphic sections, proving that this curve is rigid in the threefold.

Using the curve (3.22), we now construct the gauge flux:

$$
\mathcal{F}_{D_{1}}=\frac{1}{2} D_{1}-B+\mathcal{C}-s=\mathcal{C}-s,
$$

where we will choose the B-field $B$ such that $B-\frac{1}{2} D_{1}=0$ to cancel the Freed-Witten flux $\frac{D_{1}}{2}$. With abuse of notation, in eq. (3.23) we call $\mathcal{C}$ the two-form Poincaré dual to the curve (3.22) in $D_{1} . s$ is the pull-back of a threefold two-form, i.e. its dual cycle is the intersection of one equation $P_{s}=0$ with (3.12) and $u_{1}=0$. Deforming the $D_{1}$ equation, $P_{s}=0$ is not modified. We will choose $s$ such that the two-cycle dual to this flux is trivial in the CY threefold. This corresponds to the flux being orthogonal to the two-forms that are pulled back from the CY threefold. This is required in order to prevent chiral matter generated by the flux. $s$ is by construction of type $(1,1)$. So the condition $\mathcal{F}_{D_{1}}^{2,0}=0$ is equivalent to $\mathcal{C}^{2,0}=0$. Requiring that $\mathcal{C}$ remains of type $(1,1)$ as $D_{1}$ is deformed, is the same as requiring that the curve (3.22) is contained in the deformed divisor $u_{1}+\zeta_{2} u_{2}+\zeta_{3} u_{3}=0$. But we see that this happens only if $\zeta_{2}=\zeta_{3}=0$, i.e. the deformation moduli are fixed by this flux.

Let us determine the homology class of $s$ in the CY. ${ }^{9}$ We want that $[s]=\left.[\mathcal{C}]\right|_{C Y}$, such that $\mathcal{F}_{D_{1}}$ is trivial in the $\mathrm{CY}$. The class of $\mathcal{C}$ in the ambient space is

$$
[\mathcal{C}]=D_{y} \cdot D_{x} \cdot D_{1}=\frac{1}{2}[C Y] \cdot D_{x} \cdot D_{1}=[C Y] \cdot\left(3 D_{1}+D_{z}\right) \cdot D_{1} .
$$

So $[s]=\left.[\mathcal{C}]\right|_{C Y}=\left(3 D_{1}+D_{z}\right) \cdot D_{1}$.

Now we compute the self-intersection $\mathcal{F}_{D_{1}}^{2}$, that enters into the D3-charge of the flux

$$
-\frac{1}{2} \int_{D_{1}} \mathcal{F}_{D_{1}}^{2}=-\frac{1}{2} \int_{D_{1}}(\mathcal{C}-s) \cdot(\mathcal{C})=-\frac{1}{2} \int_{D_{1}}\left(\mathcal{C}^{2}-s^{2}\right)
$$

where we have used the fact that $\mathcal{F}_{D_{1}}$ is orthogonal to every pulled back two-form (and then also to $s$ ). The only difficult part to compute is $\mathcal{C}^{2}$. To do this we apply the following relation:

$$
\left.\mathcal{C} \cdot \mathcal{C}\right|_{D_{1}}=\int_{\mathcal{C}} \mathcal{C}=\int_{\mathcal{C}} c_{1}\left(\left.N\right|_{\mathcal{C} \subset D_{1}}\right)
$$

where we have used that the normal bundle of $\mathcal{C}$ in $D_{1}$ is a line bundle whose first Chern class is given by the class of the curve $\mathcal{C}$ in $D_{1}$. The class $c_{1}\left(\left.N\right|_{\mathcal{C} \subset D_{1}}\right)$ can be computed via the following exact sequence:

$$
\left.\left.\left.0 \rightarrow N\right|_{\mathcal{C} \subset D_{1}} \rightarrow N\right|_{\mathcal{C} \subset Y_{4}} \rightarrow N\right|_{D_{1} \subset Y_{4}} \rightarrow 0 .
$$

\footnotetext{
${ }^{9}$ In the case we are considering, two cycles of the Calabi-Yau threefold are homologous if their pushforward in the ambient fourfold are homologous.
} 
From this we get that $c_{1}\left(\left.N\right|_{\mathcal{C} \subset D_{1}}\right)=c_{1}\left(\left.N\right|_{\mathcal{C} \subset Y_{4}}\right)-c_{1}\left(\left.N\right|_{D_{1} \subset Y_{4}}\right)$. The classes on the right hand side can be easily determined by the fact that the objects involved are complete intersections in a toric space:

$$
\begin{aligned}
c_{1}\left(\left.N\right|_{\mathcal{C} \subset D_{1}}\right) & =c_{1}\left(\left.N\right|_{\mathcal{C} \subset Y_{4}}\right)-c_{1}\left(\left.N\right|_{D_{1} \subset Y_{4}}\right) \\
& =\left(D_{y}+D_{x}+D_{1}\right)-\left(2 D_{y}+D_{1}\right)=D_{x}-D_{y} \\
& =-3 D_{1}-D_{z} .
\end{aligned}
$$

Then

$$
\begin{aligned}
\left.\mathcal{C} \cdot \mathcal{C}\right|_{D_{1}} & =\int_{\mathcal{C}} c_{1}\left(\left.N\right|_{\mathcal{C} \subset D_{1}}\right)=\int_{Y_{4}}[\mathcal{C}] \cdot c_{1}\left(\left.N\right|_{\mathcal{C} \subset D_{1}}\right)=\int_{Y_{4}} D_{y} \cdot D_{x} \cdot D_{1} \cdot\left(-3 D_{1}-D_{z}\right) \\
& =\int_{X_{3}} \frac{1}{2}\left(6 D_{1}+2 D_{z}\right) \cdot D_{1} \cdot\left(-3 D_{1}-D_{z}\right)=-\int_{X_{3}} D_{1} \cdot\left(6 D_{1} \cdot D_{z}+D_{z}^{2}\right)=-3
\end{aligned}
$$

(Using analogous techniques, one can also compute $c_{1}(\mathcal{C})$ and prove that $\mathcal{C}$ is indeed a $\mathbb{P}^{1}$.)

More easily, we can compute

$$
\int_{D_{1}} s^{2}=\int_{X_{3}} D_{1} \cdot\left(3 D_{1}+D_{z}\right)^{2}=3 .
$$

We are ready to compute the D3-charge induced by this flux:

$$
-\frac{1}{2} \int_{D_{1}} \mathcal{F}_{D_{1}}^{2}=-\frac{1}{2} \int_{D_{1}}\left(\mathcal{C}^{2}-s^{2}\right)=3 .
$$

We will switch on this flux along all the 24 branes making up the $\operatorname{Sp}(24)$ stack, and its image along the 24 image branes. It is easy to prove that, as expected, $\mathcal{F}_{D_{1}}^{\prime} \equiv \mathcal{C}^{\prime}-s^{\prime}=$ $-(\mathcal{C}-s)=-\mathcal{F}_{D_{1}}$, where $\mathcal{C}^{\prime}$ is given by the equations in (3.22) with the substitution $\xi \mapsto-\xi$ and the class of $s^{\prime}$ is such that $\mathcal{F}_{D_{1}}^{\prime}$ is trivial in the CY $X_{3}$. This (diagonal) flux breaks the $\mathrm{Sp}(24)$ gauge group to $\mathrm{U}(24)$ (the $\mathrm{U}(1)$ factor remains massless, because of the triviality of the flux in $X_{3}$ ).

In conclusion, the D3-charge of the gauge flux needed to rigidify all the D7-branes in the $\operatorname{Sp}(24)$ stack is

$$
Q_{D 3}^{\mathcal{F}_{D_{1}}}=-\frac{24}{2} \int_{D_{1}} \mathcal{F}_{D_{1}}^{2}-\frac{24}{2} \int_{D_{1}} \mathcal{F}_{D_{1}}^{\prime 2}=-2 \times \frac{24}{2} \int_{D_{1}} \mathcal{F}_{D_{1}}^{2}=144
$$

\subsection{Avoiding D-terms and zero-modes from matter fields}

In section 3.2, we introduced D7-brane stacks on the divisors $D_{1}$ and $D_{5}$. If the branes carry non-zero flux, we have to worry about possible charged matter fields arising at the intersection of the two D7-brane stacks or from the D7-brane bulk spectrum. These zero modes might force the contribution to the superpotential from gaugino condensation to be zero. These problematic fluxes also generate Kähler moduli dependent Fayet-Iliopoulos (FI) terms $\xi_{i}[21,75,76]$. This would introduce a D-term potential for the Kähler moduli. However, the method of Kähler uplifting which we use requires a pure F-term potential.

In the following, we show that additional zero-modes and D-terms can be avoided for an appropriate choice of gauge flux $F$ on the branes wrapping the divisors $D_{1}$ and $D_{5}^{\text {fix }}$. 
The gauge flux $F$ combines with the pull-back of the bulk B-field on the wrapped four-cycle to give the gauge invariant field strength

$$
\mathcal{F}=F-B
$$

The number of additional zero modes and the Kähler moduli dependent Fayet-Iliopoulos terms appearing in D-terms are given by integrals of the form

$$
\int_{D_{i}} \mathcal{F}_{D_{i}} \wedge D=\int_{X_{3}} D \wedge D_{i} \wedge \mathcal{F}_{D_{i}}
$$

where $D$ is an arbitrary divisor in the threefold $X_{3}$. If it is possible to choose the fluxes $\mathcal{F}_{D_{1}}$ and $\mathcal{F}_{D_{5}^{\mathrm{fix}}}$ such that eq. (3.34) vanishes for $i=1,5$ these fluxes do not have any problematic consequences. In particular, an integral such as (3.34) vanishes if the flux $\mathcal{F}_{D_{i}}$ is orthogonal to the two-forms of $D_{i}$ that are pull-backed from the Calabi-Yau threefold $X_{3}$.

When turning on gauge flux one has to make sure that the Freed-Witten anomaly $[47,48]$ is canceled, i.e. the gauge flux on a brane wrapping divisor $D$ has to satisfy

$$
F+\frac{c_{1}(D)}{2} \in H^{2}\left(X_{3}, \mathbb{Z}\right)
$$

If the divisor $D$ is non-spin, its first Chern class $c_{1}(D)$ is odd and $F$ cannot be set to zero. On the other hand, the expression appearing in the physical quantities is the gauge invariant flux eq. (3.33). By choosing the B-field appropriately, one can make this invariant flux equal to zero. For a set of D7-brane stacks wrapping non-intersecting divisors, the global B-field can be chosen such that the pull-back on all such divisors make $\mathcal{F}=0$ for all the stacks. However, for intersecting stacks this is not possible in general. We now prove that our case is not generic in this respect: We can choose the B-field such that both $\mathcal{F}_{D_{1}}=0$ and $\mathcal{F}_{D_{5}^{\mathrm{fix}}}=0$.

The cancellation of the Freed-Witten anomaly is always satisfied if the flux that is switched on is given by

$$
F=f_{1} D_{1}+f_{5} D_{5}^{\text {fix }}+\frac{D}{2} \quad \text { with } \quad f_{1}, f_{5} \in \mathbb{Z},
$$

where we have used that $D_{1}$ and $D_{5}^{\text {fix }}$ form a basis of $H^{2}\left(X_{3}, \mathbb{Z}\right)$ and that $c_{1}(D)=-D$ in a Calabi-Yau threefold. We will only turn on diagonal fluxes on the stacks wrapping $D_{1}$ and $D_{5}^{\mathrm{fix}}$, i.e. the flux on each brane of a stack is the same (and the opposite on all the image branes). If we took non-diagonal fluxes into account this should be reflected by an additional index, for example $F^{\alpha}$ where $\alpha$ enumerates the branes.

As mentioned above we can use the B-field to fix one of the gauge fluxes $\mathcal{F}_{D_{1}}$ or $\mathcal{F}_{D_{5}^{\text {fix }}}$ to zero. In particular the Freed-Witten gauge flux, i.e. $\frac{D_{i}}{2}$, cannot be cancelled by an integral shift and then a half-integrally quantized $B$-field is needed. We choose $B=\frac{D_{1}}{2}$ (up to an integral two-form), so that choosing $F_{D_{1}}=\frac{D_{1}}{2}$ (up to the same integral two-form) one trivially realizes $\mathcal{F}_{D_{1}}=0$. The chosen B-field leads the gauge invariant flux on $D_{5}^{\text {fix }}$ to be $\mathcal{F}_{D_{5}^{\mathrm{fix}}}=F_{D_{5}^{\mathrm{fix}}}-\frac{D_{1}}{2}$. We will now show that $F_{D_{5}^{\mathrm{fix}}}$ can be tuned such that the vanishing of eq. (3.34) can also be accomplished for $D_{i}=D_{5}^{\text {fix }}$. In particular, we will show that the 
pull-back of $\frac{D_{5}^{\mathrm{fix}}}{2}-\frac{D_{1}}{2}$ on $D_{5}$ is zero. ${ }^{10}$ Let us see this in detail. After the given choice of the B-field, the gauge-invariant field strengths are given as

$$
\begin{aligned}
\mathcal{F}_{D_{1}} & =0 \\
\mathcal{F}_{D_{5}^{\text {fix }}} & =\left(a-\frac{1}{2}\right) D_{1}+\left(b+\frac{1}{2}\right) D_{5}^{\text {fix }} \quad \text { with } \quad a, b \in \mathbb{Z} .
\end{aligned}
$$

Now it is easy to see that for the choice of fluxes

$$
a=2+3 b \quad \Rightarrow \quad \mathcal{F}_{D_{5}^{\mathrm{fix}}}=\frac{1}{2}(1+2 b)\left(3 D_{1}+D_{5}^{\mathrm{fix}}\right),
$$

the pull-back of $\mathcal{F}_{D_{5}^{\text {fix }}}$ on $D_{5}^{\text {fix }}$ becomes trivial since

$$
\int_{X_{3}} D_{5}^{\text {fix }} \wedge \mathcal{F}_{D_{5}^{\text {fix }}} \wedge D \propto \int_{X_{3}} D_{5}^{\text {fix }} \wedge\left(3 D_{1}+D_{5}^{\text {fix }}\right) \wedge D=0
$$

for an arbitrary divisor $D=k_{1} D_{1}+k_{5} D_{5}^{\text {fix }} \in H^{2}\left(X_{3}, \mathbb{Z}\right)$. In the last equality in eq. (3.39) we have used the triple intersections of $X_{3}$, eq. (3.9). Hence, we have shown that additional zero-modes as well as D-terms can be avoided by tuning the gauge flux on the brane stack on $D_{5}$.

\subsection{D3 tadpole cancellation condition}

The D3 tadpole has to cancel for consistency. The compactification ingredients that induce a D3 charge are the (fluxed) D7-branes, the O7-planes, the D3-branes, the O3-planes and the RR and NS field strengths $F_{3}$ and $H_{3}$. The RR and NS fluxes and the D3-branes have a positive contribution:

$$
Q_{D 3}^{F_{3}, H_{3}}=\frac{1}{2} \int_{X_{3}} F_{3} \wedge H_{3}, \quad Q_{D 3}\left(N_{D 3} \times D 3\right)=N_{D 3} .
$$

In our case we do not have O3-planes, while we have O7-planes. Each O7-plane contributes negatively by

$$
Q_{D 3}^{O 7}=-\frac{\chi(O 7)}{6}
$$

In our construction we have two O7-planes (see eq. (3.5)), whose D3-charge sum up to

$$
Q_{D 3}^{O 7 s}=Q_{D 3}^{O 7_{u_{5}}}+Q_{D 3}^{O 7_{Q}}=-\frac{\chi\left(O 7_{u_{5}}\right)}{6}-\frac{\chi\left(O 7_{Q}\right)}{6}=-\frac{1}{6}(3+549)=-92,
$$

where we used $\chi\left(O 7_{u_{5}}\right)=3$ and $\chi\left(O 7_{Q}\right)=549$.

A stack of $N_{i}$ D7-branes and their $N_{i}$ images wrapping a divisor $D_{i}$ contributes to the total D3 charge positively via the gauge flux and negatively via a geometric contribution:

$$
Q_{D 3}^{D 7}\left(D_{i}\right)=2 N_{i}\left(-\frac{1}{2} \int_{D_{i}} \mathcal{F}_{D_{i}} \wedge \mathcal{F}_{D_{i}}-\frac{\chi\left(D_{i}\right)}{24}\right)
$$

\footnotetext{
${ }^{10}$ In principle, one could also try to choose the B-field such that $\mathcal{F}_{D_{5}^{\text {fix }}}=0$ (i.e. $B=\frac{D_{5}^{\text {fix }}}{2}$ ), instead of $\mathcal{F}_{D_{1}}=0$. The problem with this choice is that the pull-back of $\frac{D_{1}}{2}-\frac{D_{5}^{\text {fix }}}{2}$ on $D_{1}$ is not zero.
} 
where the overall factor two comes from sum over the stack and its image stack which have the same D3 charge. For the brane-stacks on $D_{1}$ and $D_{5}^{\text {fix }}$ described in section 3.2 , we obtain the following D3 tadpole:

$$
Q_{D 3}^{\text {stacks }}=-\frac{2 N_{1}}{2} \int_{D_{1}}(\mathcal{C}-s)^{2}-2 N_{1} \frac{\chi\left(D_{1}\right)}{24}-2 N_{5} \frac{\chi\left(D_{5}^{\mathrm{fix}}\right)}{24}=144-3-72=69,
$$

where we have used $N_{1}=24, N_{5}=12$, eq. (3.32) for the D3-charge of $\mathcal{F}_{D_{1}}$ and the results of table 3 .

The Whitney brane, defined by the equation $\eta^{2}-\xi^{2} \chi=0$, has a singular world volume. Thus we have to compute its contribution to the D3-tadpole indirectly. We use the fact that the Whitney brane wrapping a divisor class $D_{W}=2 D_{P}$ can be seen as the recombination of a brane wrapping the invariant divisor $D_{P}$ with the image brane wrapping the same divisor class $D_{P}$. On this brane the flux is $\mathcal{F}_{D_{P}}=\frac{1}{2} D_{P}-S-B$, while on the image brane, it is given by $-\mathcal{F}_{D_{P}}$. Here $B$ is the $B$-field and $S$ an arbitrary integral class. After recombination only a flux that is trivial along the CY survives (see $[53,65]$ ). In the recombination process, the RR-charges do not change. Hence, we can compute the D3-charge in the more tractable brane/image-brane situation, as done in [37, 65]:

$$
Q_{D 3}^{W}=-\int_{D_{P}} \mathcal{F}_{D_{P}}^{2}-\frac{\chi\left(D_{P}\right)}{12}
$$

In order for the Whitney brane to be holomorphic (supersymmetric), $D_{P}$ must be such that $D_{P}-[O 7]>0$ (i.e. it can be described by the vanishing of a holomorphic equation). The choice of $S$ in not completely arbitrary. It must satisfy the following constraints, in order for the Whitney brane to be stable against splitting:

$$
\frac{[O 7]}{2} \leq S+B \leq D_{P}-\frac{[O 7]}{2}
$$

We will compute the charge using the description of $X_{3}$ as a hypersurface in the ambient space $X_{4}^{\text {amb,123 }}$ (3.10). The equation of the Whitney brane, after factorizing the $\mathrm{Sp}(24)$ and the $\mathrm{SO}(24)$ stacks is given by $\hat{\eta}_{12,4}^{2}-\xi^{2} \hat{\chi}_{6,2}$. In our example the B-field is fixed by the vanishing of $\mathcal{F}_{D_{1}}$ to be $B=\frac{D_{1}}{2}$. The class of the Whitney brane is $D_{W}=2 D_{P}=$ $2\left(12 D_{1}+4 D_{z}\right)$, while $[O 7]=D_{y}=9 D_{1}+3 D_{z}$. If we take $S=f_{1} D_{1}+f_{z} D_{z}\left(f_{1}, f_{z} \in \mathbb{Z}\right)$, the constraints (3.46) translate to $4 \leq f_{1} \leq 7$ and $\frac{3}{2} \leq f_{z} \leq \frac{5}{2}$, i.e. $f_{1}=4,5,6,7$ and $f_{z}=2$.

Let us compute the Whitney brane D3-charge. The Euler number of $D_{P}$ is

$$
\chi\left(D_{P}\right)=\int_{X_{3}} D_{P}^{3}+D_{P} \cdot c_{2}\left(X_{3}\right)=984
$$

while

$$
\int_{D_{P}} \mathcal{F}_{D_{P}}^{2}=\int_{X_{3}} D_{P}\left(\frac{D_{P}}{2}-\frac{D_{1}}{2}-\left(f_{1} D_{1}+f_{z} D_{z}\right)\right)^{2}=-\left(2 f_{1}-11\right)^{2}
$$

Inserting these expressions in (3.45) and using the possible values for $f_{1}$, we obtain two possible results for the charge of the Whitney brane, i.e. $Q_{D 3}^{W}=-81$ and $Q_{D 3}^{W}=-73$. 
Taking into account the contribution from the O7-planes and the D7-brane stacks, eq. (3.42) and (3.44), and the negative contribution from the Whitney brane, we obtain the following total D3-brane charge from our brane configuration:

$$
Q_{D 3}^{\text {tot }}=Q_{D 3}^{\mathrm{O} 7 \mathrm{~s}}+Q_{D 3}^{\mathrm{stacks}}+Q_{D 3}^{W}=\left\{\begin{array}{lll}
-104 & \text { for } & Q_{D 3}^{W}=-81 \\
-96 & \text { for } & Q_{D 3}^{W}=-73
\end{array} .\right.
$$

At this point, we have a fully consistent picture of the D brane and gauge flux setup in our threefold $X_{3}$ that ensures that gaugino condensation from the divisors $D_{1}$ and $D_{z}$ contributes to the superpotential of the four dimensional $\mathcal{N}=1$ effective supergravity. In particular, we have overcome the issues discussed in section 2. Before we discuss moduli stabilization in a de Sitter vacuum in section 6 , let us have a look at the brane and flux configuration from the F-theory point of view in the following section 4.

\section{The F-theory perspective of $\mathbb{C P}_{11169}^{4}[18]$}

In this section, we revisit some results of the previous section by using the F-theory language. We first discuss the D7-brane configuration in F-theory, according to table 1. We consider the Calabi-Yau fourfold that is an elliptic fibration over the base manifold $B_{3}$ defined in eq. (3.6). We find that enforcing an $\operatorname{Sp}(24)$ singularity on the divisor $\hat{D}_{1}$ of $B_{3}$ forces us to impose an $\mathrm{SO}(24)$ singularity on $\hat{D}_{5}$. This agrees with the type IIB perspective discussed in section 3.2 .

Let us see this in detail. The Tate polynomials are sections of $\bar{K}^{i}$ which we denote as $a_{i}=A_{9 i, 2 i}$. By realizing an $\operatorname{Sp}(24)$ gauge group on $D_{1}$ we need the following factorization of the Tate polynomials:

$$
\begin{aligned}
& a_{1}=A_{9,2}=u_{5} A_{9,1} \\
& a_{2}=A_{18,4}=u_{5} A_{18,3} \\
& a_{3}=u_{1}^{24} A_{3,6}=u_{1}^{24} u_{5}^{6} A_{3,0}, \\
& a_{4}=u_{1}^{24} A_{12,8}=u_{1}^{24} u_{5}^{6} A_{12,2}, \\
& a_{6}=u_{1}^{48} A_{6,12}=u_{1}^{48} u_{5}^{11}\left(u_{4} A_{0,0}+u_{5} A_{6,0}\right),
\end{aligned}
$$

where on the r.h.s. of (4.1) we made the $u_{5}$ factorization explicit. Comparing with table 1 , we see that the $a_{i}$ in eq. (4.1) are compatible both with an $\mathrm{SO}(4 \cdot 5+3=23)$ and an $\mathrm{SO}(4 \cdot 5+4=24)$ singularity on $\hat{D}_{5}$. For generic polynomials $A_{p, q}$, the first is realized. The $\mathrm{SO}(24)$ is present when the polynomial $A_{18,3} X^{2}+A_{12,2} X+\left(u_{4} A_{0,0}+u_{5} A_{6,0}\right)$ factorizes modulo $u_{5}$ (i.e. modulo a polynomial that is divided by $u_{5}$ ). This happens if $A_{0,0}=0$, that is the choice we have done in the perturbative type IIB limit in section $3.2\left(\chi=4 a_{6}+a_{3}^{2}\right)$.

Now we consider the fourfold where the $\operatorname{Sp}(24)$ singularity is resolved along the lines described in appendix B.1. The resolution introduces a set of new divisors, the exceptional divisors $E_{2 i-1}(i=1, \ldots, N)$, that are $\mathbb{P}^{1}$ fibrations over the surface in the base $B_{3}$ where the fiber degenerates. From the F-theory point of view, the gaugino condensation contribution to the superpotential is generated by M5-instantons wrapping the exceptional divisors 
that resolve the corresponding singularity [77]. In the presence of fluxes, the necessary condition for an M5-instanton wrapping a divisor $D$ to contribute to the superpotential is that $\chi_{0}(D) \geq 1$, which is the known modification of the condition $\chi_{0}(D)=1$ without fluxes $[69,78]$.

In appendix B.1, we derive the following formula for the arithmetic genus of the exceptional divisors

$$
\begin{aligned}
\chi_{0}\left(E_{2 i-1}\right) & =\frac{1}{6} \int_{B_{3}} \hat{D} \wedge\left[c_{2}\left(B_{3}\right)+\bar{K} \wedge \bar{K}+2 \hat{D} \wedge \hat{D}\right]=\chi_{0}(D) ; i=1, \ldots, N-1, \\
\chi_{0}\left(E_{2 N-1}\right) & =\frac{1}{12} \int_{B_{3}} \hat{D} \wedge\left[c_{2}\left(B_{3}\right)+(\bar{K}-\hat{D}) \wedge(\bar{K}-2 \hat{D})\right]=\chi_{0}(\hat{D}),
\end{aligned}
$$

where $\hat{D}$ is the divisor $\left\{p_{\hat{D}}=0\right\}$ on the base manifold $B_{3}$ where the singularity sits and $D$ is its double cover in $X_{3}$.

In our example we imposed an $\operatorname{Sp}\left(N_{1}=24\right)$ singularity on the divisor $\hat{D}_{1}$. Inserting

$$
\bar{K}=9 \hat{D}_{1}+2 \hat{D}_{5}, \quad c_{2}\left(B_{3}\right)=21 \hat{D}_{1}^{2}+12 \hat{D}_{1} \hat{D}_{5}+\hat{D}_{5}^{2},
$$

and the base intersections eq. (3.8) into eq.s (4.2) we find

$$
\chi_{0}\left(E_{2 i-1}\right)=3 \text { for } i=1, \ldots, N_{1}-1 \quad \text { and } \quad \chi_{0}\left(E_{2 N_{1}-1}\right)=1 .
$$

We see that all of them satisfy the necessary condition for an M5-instanton to contribute to the superpotential in the presence of fluxes. This agrees with what we found in type IIB language, where we have seen that this actually happens, i.e. switching on a proper gauge flux fixes the deformation on the wrapped divisor, leading to the possibility of having gaugino condensation.

Let us summarize the F-theory analysis. The D-brane configuration is equivalent to what we find in section 3.2 in the IIB picture. Furthermore, we calculated the arithmetic genus of the exceptional divisors that correspond to the resolution of the $\operatorname{Sp}(N)$ singularity for a general base manifold. For our example, the results show that one has to switch on gauge flux so that the divisor can carry a non-vanishing contribution from gaugino condensation to the superpotential. The corresponding type IIB analysis was done in section 3.3.

\section{Moduli stabilization in the large volume limit}

Let us define the four dimensional effective theory. In the absence of $D$-terms the scalar potential is completely determined by the Kähler potential $K$ and the superpotential $W$ via

$$
V=e^{K}\left(K^{\alpha \bar{\beta}} D_{\alpha} W \overline{D_{\beta} W}-3|W|^{2}\right),
$$

where $D_{\alpha} W=W_{\alpha}+K_{\alpha} W$, with subscripts $\alpha, \beta$ denoting the derivative w.r.t. complex scalar moduli fields $\phi_{\alpha}$. These are the Kähler moduli $T_{i}, i=1, \ldots, h^{1,1}$, the dilaton $S$ and the complex structure moduli $U_{a}, a=1, \ldots, h^{2,1}$.

The Kähler potential is a real function of these moduli [79]:

$$
K=-2 \ln \left(\mathcal{V}\left(T_{i}, \bar{T}_{i}\right)+\frac{1}{2} \hat{\xi}(S, \bar{S})\right)-\ln (S+\bar{S})-\ln \left(-i \int \bar{\Omega}\left(\bar{U}_{a}\right) \wedge \Omega\left(U_{a}\right)\right),
$$


where $\mathcal{V}$ denotes the volume and $\Omega$ the holomorphic three-form of the threefold while the dilaton dependent $\alpha^{\prime}$ correction is given as

$$
\hat{\xi}(S, \bar{S})=-\frac{\zeta(3) \chi}{4 \sqrt{2}(2 \pi)^{3}}(S+\bar{S})^{3 / 2},
$$

with $\chi$ being the Euler number of the threefold and $\zeta(3) \simeq 1.202$.

The holomorphic superpotential

$$
W=W_{0}\left(S, U_{a}\right)+\sum_{i} A_{i}\left(S, U_{a}\right) e^{-a_{i} T_{i}},
$$

is the sum of a tree-level and a non-perturbative contribution. In the model we consider, the non-perturbative exponential term arises from gaugino condensation in pure non-Abelian 4D $\mathcal{N}=1$ super-Yang-Mills theories on stacks of D7-branes wrapping appropriate four-cycle divisors of the Calabi-Yau threefold. Here $A_{i}\left(S, U_{a}\right)$ denotes the one-loop determinant, and $a_{i}=2 \pi / N_{i}$ where $N_{i}$ is the Coxeter number of the corresponding gauge group singularity in F-theory. The perturbative term consists of the flux induced GukovVafa-Witten superpotential [51]

$$
W_{0}\left(S, U_{a}\right)=\frac{1}{2 \pi} \int\left(F_{3}-S H_{3}\right) \wedge \Omega\left(U_{a}\right)
$$

with $S=s+i \sigma, s=1 / g_{s}$ and $F_{3}$ and $H_{3}$ the RR and NS three-form field strength respectively.

As discussed in much recent work (see [5] for the original seminal result, and [1-3] for recent review), turning on primitive imaginary self-dual (ISD) three-form fluxes generically leads to a supersymmetric stabilization of all complex structure moduli and the axio-dilaton at an isolated supersymmetric extremum in moduli space. The flux induced superpotential is independent of the Kähler moduli, which implies

$$
K^{i \bar{\jmath}} D_{i} W_{0} \overline{D_{j} W_{0}}=3\left|W_{0}\right|^{2}
$$

and thus a no-scale scalar potential for the complex structure moduli and the axio-dilaton

$$
V_{f l u x}=e^{K}\left(K^{S \bar{S}}\left|D_{S} W_{0}\right|^{2}+K^{a \bar{b}} D_{a} W_{0} \overline{D_{b} W_{0}}\right) .
$$

This potential is positive semi-definite. Hence, every flux-induced isolated supersymmetric extremum for the axio-dilaton and the complex structure moduli has a positivedefinite mass matrix, and is a true local minimum. For $W=W_{0}$ the Kähler moduli are flat directions. Volume moduli stabilization may now proceed by several methods. KKLT generates SUSY AdS minima for the volumes from non-perturbative effects balancing off a small $W_{0}$. On the other hand, in LVS an interplay between non-perturbative and perturbative effects generates non-SUSY AdS vacua at exponentially large volumes, or AdS/dS vacua at largish volumes in the Kähler uplifting scenario studied here. All these stabilization methods necessarily proceed via breaking the no-scale structure. The question of full stability of a given scenario or concrete model rests then on the effect of the no-scale 
breaking contributions from volume stabilization to the mass matrix of the complex structure moduli and the axio-dilaton. One can show for both the LVS and the Kähler uplifting scenario, that all no-scale breaking terms are suppressed by an extra inverse power $1 / \mathcal{V}$ of the volume compared to the flux-induced piece above $[8,24]$. As the flux-induced piece is positive semi-definite and $\mathcal{O}\left(1 / \mathcal{V}^{2}\right)$, any negative term must come from no-scale breaking contributions which are $\mathcal{O}\left(1 / \mathcal{V}^{3}\right)$. Hence, any small shift of $S$ or one of the $U_{a}$ will see a positive $\mathcal{O}\left(1 / \mathcal{V}^{2}\right)$ increase in the scalar potential overwhelming any possible decreasing $\mathcal{O}\left(1 / \mathcal{V}^{3}\right)$ contribution from Kähler moduli stabilization. The only condition for this automatic separation of scales to work is stabilization of the over-all volume at largish values in the first place.

Thus, any choice of flux producing an isolated SUSY extremum $D_{S} W=D_{U_{a}} W=0$ will be a minimum of the full potential once the LVS or Kähler uplifting generate a local minimum for the Kähler moduli at large volumes. Thus there is no need for a detailed model-by-model calculation of flux-induced mass matrix for the complex structure moduli and the axio-dilaton (a task practically unfeasible for typical values $h^{2,1}=\mathcal{O}(100)$ ).

\section{A fully stabilized de Sitter vacuum of $\mathbb{C P}_{11169}^{4}[18]$}

In this section, we show that all geometric moduli of $\mathbb{C P}_{11169}^{4}[18]$ can be stabilized in a metastable de Sitter vacuum. The stabilization of the two Kähler moduli via the interplay of non-perturbative effects in the superpotential and $\alpha^{\prime}$ corrections in the Kähler potential will be discussed in section 6.1. The analysis will single out values for the flux superpotential $W_{0}$, the string coupling $g_{s}$ and the one-loop determinant of gaugino-condensation $A$ which have to be realized to construct a de Sitter vacuum. We will demonstrate in section 6.2 that the values of these parameters can be provided by explicitly solving a two dimensional subspace of the complex structure moduli space of $X_{3}$, hence providing an explicit construction of a de Sitter vacuum.

\subsection{Kähler uplifted de Sitter vacua}

Realizing the brane and gauge flux setup discussed in sections 3 and 4 there are two Kähler moduli $T_{1}=\tau_{1}+i \zeta_{1}$ and $T_{2}=\tau_{2}+i \zeta_{2}$ whose real components correspond to the volumes of the divisors $D_{1}$ and $D_{5}$, i.e. $\tau_{1} \equiv \mathcal{V}_{1}$ and $\tau_{2} \equiv \mathcal{V}_{5}$. The volume form of the three-fold eq. (3.14) leaves us with the following Kähler potential for the Kähler moduli:

$$
K=-2 \log \left[\frac{1}{\sqrt{12}}\left(\left(T_{1}+\bar{T}_{1}\right)+\frac{1}{3}\left(T_{2}+\bar{T}_{2}\right)\right)^{3 / 2}-\frac{1}{18}\left(T_{2}+\bar{T}_{2}\right)^{3 / 2}+\frac{1}{2} \hat{\xi}(S, \bar{S})\right],
$$

where $\hat{\xi}(S, \bar{S})$ is given in eq. (5.3) with $\chi=2(2-272)=-540$.

The superpotential arising from gaugino condensation of a $\mathrm{SU}(24)$ and $\mathrm{SO}(24)$ pure Super Yang Mills (SYM) on the D7-brane stacks wrapping $D_{1}$ and $D_{5}$ is given as

$$
W=W_{0}+A_{1} e^{-\frac{2 \pi}{24} T_{1}}+A_{2} e^{-\frac{2 \pi}{22} T_{2}},
$$


i.e. $a_{1}=2 \pi / 24$ and $a_{2}=2 \pi / 22$. We know that the one-loop determinants of the two gaugino condensates are non-zero since $D_{5}$ is rigid and $D_{1}$ has been 'rigidified' by flux. ${ }^{11}$ However, it is not know how to calculate the dependence of $A_{1}$ and $A_{2}$ on the complex structure moduli and the axio-dilaton. After stabilizing these moduli, $A_{1}$ and $A_{2}$ (as well as $\left.W_{0}\right)$ are flux-dependent parameters and can be treated as constant in the Kähler moduli stabilization process. In general, they will be of the same order as the VEVs of the complex structure moduli. Since there is a large number of flux parameters due to $h^{2,1}=272$, it seems a reasonable assumption that one should be able to use the freedom in this sector to mildly tune $A_{1}$ and $A_{2}$ to a value desired for the stabilization of the Kähler moduli. Nevertheless, the unknown dependence of the one-loop determinants on the complex structure moduli remains the only point that cannot be addressed explicitly in our construction of a de Sitter vacuum.

The scalar potential of the moduli $T_{1}, T_{2}$ is

$$
V=e^{K}\left(K^{T_{i} \bar{T}_{j}}\left[W_{T_{i}} \overline{W_{T_{j}}}+\left(W_{T_{i}} \cdot \overline{W K_{T_{j}}}+c . c\right)\right]+3 \hat{\xi} \frac{\hat{\xi}^{2}+7 \hat{\xi} \hat{\mathcal{V}}+\hat{\mathcal{V}}^{2}}{(\hat{\mathcal{V}}-\hat{\xi})(\hat{\xi}+2 \hat{\mathcal{V}})^{2}}|W|^{2}\right) .
$$

Considering the dilaton $S$ and the complex structure moduli $U_{a}$ to be fixed by fluxes, which will be done explicitly in section 6.2, eq. (6.3) is a function $V=V\left(T_{1}, T_{2}\right)$ with parameters $W_{0}, S, A_{1}$ and $A_{2}$. A de Sitter vacuum will only be obtained for certain values of these four parameters. In the following we consider the axio-dilaton to have a real $\operatorname{VEV} s=\operatorname{Re}(S)$, i.e. the axionic component is stabilized at zero VEV.

In [24] a sufficient condition on the compactification parameters was found which ensures that a de Sitter vacuum can be constructed via the method of Kähler uplifting. The condition applies when the manifold is of the swiss-cheese type. For a one parameter model this condition reads

$$
3.65 \lesssim \frac{-27 W_{0} \hat{\xi} a^{3 / 2}}{64 \sqrt{2} \gamma A} \lesssim 3.89
$$

where $\gamma$ is related to the self-intersection number of the wrapped four cycle. For our brane setup on $\mathbb{C P}_{11169}^{4}[18]$ the volume form is only approximately of the swiss-cheese type so that the analytic results of [24] cannot be applied directly. Nevertheless, we can study the scalar potential given in eq. (6.3) numerically, searching for stable de Sitter minima for fixed numerical values of $W_{0}, S, A_{1}$ and $A_{2}$.

We find that there is indeed a subspace in this parameter space where the two Kähler moduli are stabilized in a stable de Sitter vacuum. For instance, keeping $A_{1}$ and $A_{2}$ constant we find that there is a curve in $W_{0}-S$ space that allows stable de Sitter vacua, see figure 2. Actually, it is a band rather than a curve, the lower bound of the band corresponding to Minkowski vacua and the upper bound corresponding to the minimum becoming an inflection point. For a single Kähler modulus this corresponds to the upper

\footnotetext{
${ }^{11}$ The 'rigidifying' flux on $D_{1}$ induces a correction to the gauge kinetic function of the form $T_{1} \rightarrow$ $T_{1}-\frac{1}{2 g_{s}} \int_{D_{1}} \mathcal{F}_{D_{1}} \wedge \mathcal{F}_{D_{1}}$ which is non-zero since $\int_{D_{1}} \mathcal{F}_{D_{1}} \wedge \mathcal{F}_{D_{1}}=-6$, see eq. (3.31). This can be absorbed in the one-loop determinant $A\left(S, U_{a}\right)$ since the correction does not depend on the $T_{i}$. We thank Gary Shiu for bringing this to our attention.
} 


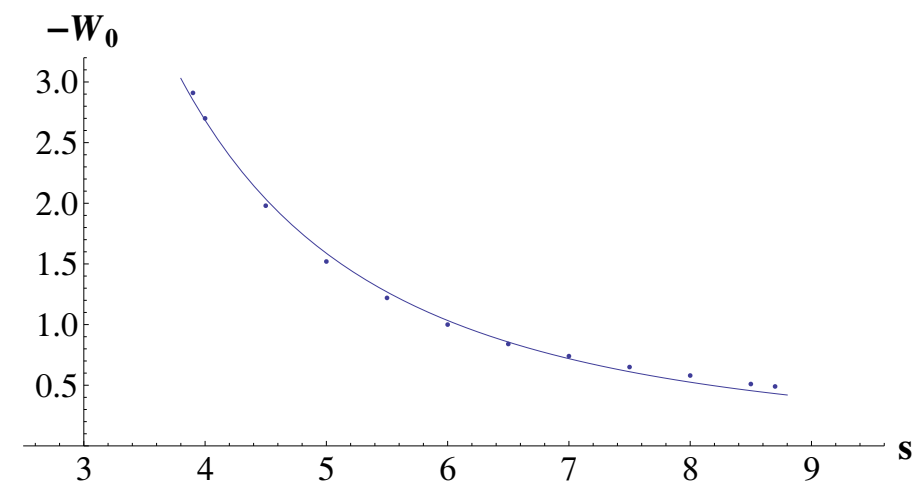

Figure 2. The points represent $W_{0}, S$ pairs that allow a stable de Sitter solution of the potential given in eq. (6.3) for $A_{1}=A_{2}=1$. The curve represents a fit $W_{0}=C_{1} s^{-C_{2}}$, with $C_{1}=70.2$ and $C_{2}=2.35$. Note that there is a small deviation from the one modulus case where $C_{2}=1.5$.

and lower bound in eq. (6.4), respectively. Since the width of the band is rather small and we are interested in vacua with a small cosmological constant, we choose to display the lower bound in figure 2 .

In the remainder of this section, let us study explicitly the following point in parameter space that realizes a de Sitter vacuum:

$$
W_{0}=0.812, \quad s=6.99, \quad A_{1}=1.11, \quad A_{2}=1.00 .
$$

The choice of numerical values in eq. (6.5) is due to the solutions we find in the complex structure sector, see section 6.2. $W_{0}$ and $s$ originate from this sector and $A_{1}$ and $A_{2}$ have to be chosen appropriately, invoking the constraint that generically they are of the same order as the VEVs of the complex structure moduli, in this case $\mathcal{O}(1)$ as we will see in section 6.2.

We can justify both treating the instanton prefactors $A_{i}$ as effective constants, and mildly tuning them. Recent work has shown [24] that for large volume the mass scale of the Kähler moduli separates from the scale of the axio-dilaton and the complex structure moduli by one inverse power of the volume. This justifies replacing the complex structure moduli by their VEVs inside the 1-loop determinants, and allows us to parametrize these prefactors as effective constants. Moreover, we can clearly dial the VEVs of the complex structure moduli by availing ourselves of the exponentially large flux discretuum, which easily accounts for a potential mild tuning of the value of the 1-loop determinants.

The phenomenology of the model (6.5) is summarized in table 4 and figure 3 . In particular, we note that the overall volume and the volume of the divisors $D_{1}$ and $D_{5}$ are stabilized at $\mathcal{O}(10-100)$. The not too large overall volume emerges from the fact that we have only realized an $N_{1}=24$ gauge group on $D_{1}$ which is actually lower than the critical gauge group rank $\sim 30$, as was discussed in section 2 . Note that we were forced to choose the rank smaller than the maximal rank $N_{\mathrm{lg}}=27$ in order to consistently incorporate the subtleties in the D7-brane configuration and construct a fully consistent model, see section 3. Since models with a larger number of Kähler moduli allow in principle larger maximal gauge group rank, one may also realize larger overall volumes in these more complicated cases. 


\begin{tabular}{|c|c|c|c|c|c|c|c|}
\hline$\left\langle T_{1}\right\rangle$ & $\left\langle T_{2}\right\rangle$ & $\langle\mathcal{V}\rangle$ & $m_{\tau_{1}}^{2}$ & $m_{\tau_{2}}^{2}$ & $m_{\zeta_{1}}^{2}$ & $m_{\zeta_{2}}^{2}$ & $m_{3 / 2}^{2}$ \\
\hline 10.76 & 12.15 & 51.97 & $5.24 \cdot 10^{-9}$ & $4.55 \cdot 10^{-8}$ & $1.13 \cdot 10^{-7}$ & $6.40 \cdot 10^{-8}$ & $4.08 \cdot 10^{-7}$ \\
\hline
\end{tabular}

Table 4. VEVs and masses of the Kähler moduli. $m_{3 / 2}^{2}=e^{K}|W|^{2}$ denotes the gravitino mass. For the calculation of the masses the prefactor $\left\langle e^{K_{\text {c.s. }}}\right\rangle \simeq 0.03$ from the complex structure moduli stabilization, see following section 6.2, has been taken into account. All masses are given in units of the Planck mass while volumes are given in units of $\alpha^{\prime}$.
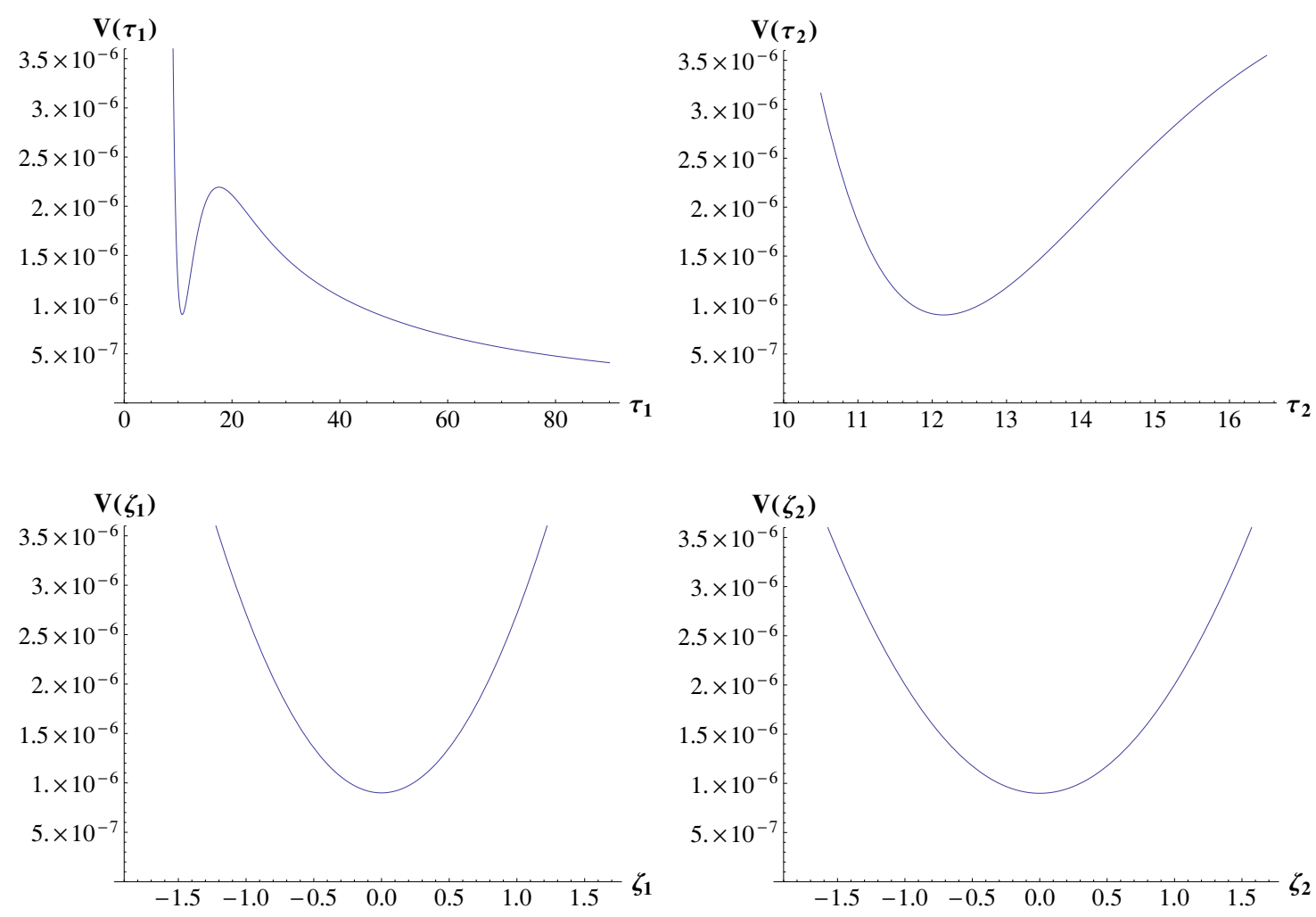

Figure 3. The scalar potential $V\left(T_{1}, T_{2}\right)$ is a function of four real scalar fields. We show $V\left(T_{1}, T_{2}\right)$ as a single valued function with the other three fields evaluated at the minimum.

The Kähler moduli are stabilized inside the Kähler cone which corresponds to $t_{1}>3 t_{5}$. Inverting the relation between the $t_{i}$ and the $\mathcal{V}_{i}$, eq. (3.14), we find $t_{1}=9.43$ and $t_{5}=1.50$ such that the Kähler cone condition is fulfilled.

\subsection{Complex structure moduli}

In this section, we study the complex structure moduli space of $X_{3}$. In the end, we will present explicit RR and NS flux choices that stabilize the dilaton and complex structure moduli supersymmetrically such that $W_{0}$ and $s$ take the values in eq. (6.5). Note that switching on RR and NS flux will not introduce Freed-Witten anomalies [48] since the fourcycles $D_{1}$ and $D_{5}^{\mathrm{fix}}$ wrapped by D7-branes have no three-cycles (as $h^{1,0}\left(D_{1}\right)=h^{1,0}\left(D_{5}^{\mathrm{fix}}\right)=$ 0 , see table 3 ). Hence, any three-form of the threefold $X_{3}$ is pulled back to zero on the 
D7-branes, making the Freed-Witten constraint $\left.H_{3}\right|_{D 7}=0$ automatically satisfied. ${ }^{12}$ Since it was shown in the previous section that the parameters (6.5) lead to a stable de Sitter vacuum in the Kähler moduli sector, $\mathbb{C P}_{11169}^{4}[18]$ will provide us with an explicit example of such a vacuum with all geometric moduli stabilized.

As was discussed in $[70,80,81]$, the 272 dimensional complex structure moduli space of $\mathbb{C P}_{11169}^{4}[18]$ is symmetric under a $\Gamma=\mathbb{Z}_{6} \times \mathbb{Z}_{18}$ action. Under $\Gamma$, only two complex structure moduli are left invariant. As was noted in [70] it is sufficient to turn on fluxes only along the six $\Gamma$-invariant three-cycles to achieve $D_{i} W=0$ for all 272 complex structure moduli, and then to find a minimum of the positive definite tree-level no-scale scalar potential $V=\sum_{i}\left|D_{i} W\right|$. This is due to the fact that, for this invariant flux, the symmetry $\Gamma$ is realized at the level of the four-dimensional effective action. Note that the restriction to flux on the $\Gamma$ invariant cycles is purely for simplicity, as the analysis of the complete 272 dimensional complex structure moduli space is practically not doable.

Once the symmetric fluxes are switched on, the Kähler potential and superpotential are

$$
\begin{aligned}
K & =-\log \left(-i \Pi^{\dagger} \cdot \Sigma \cdot \Pi\right)-\log (S+\bar{S}), \\
W_{0} & =2 \pi(f-S h) \cdot \Pi,
\end{aligned}
$$

where $\Pi^{I}$ is the vector of periods of the Calabi-Yau threefold holomorphic three-form $\Omega_{3}$ on a symplectic basis of three-cycles, $\Sigma$ is the canonical symplectic matrix and $f=$ $\left(f_{1}, \ldots, f_{6}, 0, \ldots, 0\right)$ and $h=\left(h_{1}, \ldots, h_{6}, 0, \ldots, 0\right)$ are the integer valued RR and NS flux quantum numbers (we have set to zero all the components along the $b_{3}-6$ non-invariant three-cycles.

Let us explain why this flux vector generically provides a stable minimum of all 272 complex structure moduli $[49,70]$. We first consider $D_{\tilde{U}_{i}} W_{0}=0$, where $\tilde{U}_{i}$ for $i=3, \ldots, 272$ denote the non-trivially transforming moduli under $\Gamma=\mathbb{Z}_{6} \times \mathbb{Z}_{18}$. The period vector is, at leading order, a polynomial function of the $\tilde{U}_{i}$. Furthermore, $\Pi$ cannot contain linear powers of the $\tilde{U}_{i}$, since these would not be invariant under $\Gamma$ and the period vector has to respect the symmetry of the complex structure moduli space. This information is sufficient to show

$$
W_{0, \tilde{U}_{i}}=K_{\tilde{U}_{i}}=0 \quad \text { at } \quad \tilde{U}_{i}=0 \text { for } \quad i=3, \ldots, 272,
$$

since $W_{0, \tilde{U}_{i}}$ is a polynomial function which is at least linear in the $\tilde{U}_{i}$, see eq. (6.7) and $K_{\tilde{U}_{i}}$ is a rational function which is at least linear in the numerator in the $\tilde{U}_{i}$, see eq. (6.6). Hence, $D_{\tilde{U}_{i}} W_{0}=W_{0, \tilde{U}_{i}}+K_{\tilde{U}_{i}} W_{0}=0$ at $\tilde{U}_{i}=0$ for $i=3, \ldots, 272$. This reduces the full set of conditions $D_{i} W=0 \forall i$ to the three equations

$$
\left.D_{\phi} W\right|_{\tilde{U}_{i}=0}=0 \quad \text { for } \phi=S, U_{1}, U_{2},
$$

where $U_{1}$ and $U_{2}$ are the two invariant complex structure moduli. This is equivalent to set $\tilde{U}_{i}=0$ from the beginning and study the stabilization problem for the reduced case with two complex structure moduli, as we do in the following.

\footnotetext{
${ }^{12}$ Unfortunately we are not able to check the Freed-Witten anomaly cancellation due to $H_{3}$ for the Whitney brane. In fact, due to its singular world volume, the solution of this problem for a generic Whitney brane is so far unknown.
} 
In [81], the prepotential $\mathcal{G}$ for the two complex structure moduli $U_{1} \equiv \nu_{1}+i u_{1}=\omega_{1} / \omega_{0}$ and $U_{2} \equiv \nu_{2}+i u_{2}=\omega_{2} / \omega_{0}$ was derived via mirror symmetry in the large complex structure limit to be

$$
\mathcal{G}\left(\omega_{0}, \omega_{1}, \omega_{2}\right)=\xi \omega_{0}^{2}+\frac{17 \omega_{0} \omega_{1}}{4}+\frac{3 \omega_{0} \omega_{2}}{2}+\frac{9 \omega_{1}^{2}}{4}+\frac{3 \omega_{1} \omega_{2}}{2}-\frac{9 \omega_{1}^{3}+9 \omega_{1}^{2} \omega_{2}+3 \omega_{1} \omega_{2}^{2}}{6 \omega_{0}},
$$

with $\xi=\frac{\zeta(3) \chi}{2(2 \pi i)^{3}} \simeq-1.30843 i$ determined by the Euler number $\chi$ of the Calabi-Yau.

Eq. (6.10) receives instanton corrections which are given as

$$
\begin{aligned}
\mathcal{G}_{\text {inst. }}\left(q_{1}, q_{2}\right)=\frac{1}{(2 \pi i)^{3}}( & 540 q_{1}+\frac{1215 q_{1}^{2}}{2}+560 q_{1}^{3}+3 q_{2}-1080 q_{1} q_{2}+143370 q_{1}^{2} q_{2} \\
& \left.-\frac{45 q_{2}^{2}}{2}+2700 q_{1} q_{2}^{2}+\frac{244 q_{2}^{3}}{9}+\ldots\right)
\end{aligned}
$$

with $q_{i}=\exp \left(2 \pi i \omega_{i}\right)$ and we have set $\omega_{0}=1$. The dots in eq. (6.11) denote higher powers in the $q_{i}$ which are suppressed in the large complex structure limit $\omega_{i} \gg 1$.

For a symplectic basis of three-cycles, the period vector is given by

$$
\begin{aligned}
\Pi= & \left(\partial_{\omega_{0}} \mathcal{G}, \partial_{\omega_{1}} \mathcal{G}, \partial_{\omega_{2}} \mathcal{G}, \omega_{0}, \omega_{1}, \omega_{2}\right) \\
= & \left(2 \xi+\frac{17 U_{1}}{4}+\frac{3 U_{1}^{3}}{2}+\frac{3 U_{2}}{2}+\frac{3}{2} U_{1}^{2} U_{2}+\frac{1}{2} U_{1} U_{2}^{2},\right. \\
& \left.\frac{17}{4}+\frac{9 U_{1}}{2}-\frac{9 U_{1}^{2}}{2}+\frac{3 U_{2}}{2}-3 U_{1} U_{2}-\frac{U_{2}^{2}}{2}, \frac{3}{2}+\frac{3 U_{1}}{2}-\frac{3 U_{1}^{2}}{2}-U_{1} U_{2}, 1, U_{1}, U_{2}\right) .
\end{aligned}
$$

Finally, the D3-charge induced by the RR and NS fluxes is given by

$$
Q_{D 3}^{f, h}=\frac{1}{2} h \cdot \Sigma \cdot f=66 .
$$

Our goal is to find flux quanta $f$ and $h$ that stabilize $S, U_{1}$ and $U_{2}$ supersymmetrically, i.e. $D_{\phi} W_{0}=0$ for $\phi=S, U_{1}, U_{2}$, with $\left\langle W_{0}\right\rangle$ and $\langle S\rangle$ suitable to perform the Kähler moduli stabilization in a de Sitter vacuum.

To find stationary points we apply the strategy suggested in [70]: ${ }^{13}$

- Neglecting instanton corrections to the prepotential and approximating $|\xi| \simeq$ $1.30843 \ldots$ by an approximate rational value, for instance $|\xi|=13 / 10$ we solve the system of equations

$$
0=\left(W_{0}, D_{S} W_{0}, D_{U_{1}} W_{0}, D_{U_{2}} W_{0}\right)_{\text {(no inst.) }},
$$

for the flux quanta $f$ and $h$ setting the VEVs $S, U_{1}$ and $U_{2}$ to fixed rational values. Furthermore, we require $f$ and $h$ to fulfill the tadpole constraint found in eq. (3.49), $Q_{D 3}^{f, h} \leq 104$ or $Q_{D 3}^{f, h} \leq 96$. This amounts to solving a linear equation of the form $A \cdot(f, h)=0$ with $A \in \mathbb{Q}^{8 \times 12}$ for $f$ and $h$, respecting the tadpole constraint.

\footnotetext{
${ }^{13} \mathrm{~A}$ detailed analysis of the flux solution space will be presented in [82]. Among others, one can use a recently developed method, called numerical polynomial homotopy continuation method, which can find all the stationary points of a given potential having polynomial-like nonlinearity [83].
} 


\begin{tabular}{|c|c|c|c|c|c|}
\hline$m_{u_{1}}^{2}$ & $m_{u_{2}}^{2}$ & $m_{s}^{2}$ & $m_{\nu_{1}}^{2}$ & $m_{\nu_{2}}^{2}$ & $m_{\sigma}^{2}$ \\
\hline 0.24 & $1.8 \cdot 10^{-4}$ & $5.6 \cdot 10^{-6}$ & 0.24 & $1.8 \cdot 10^{-4}$ & $5.7 \cdot 10^{-6}$ \\
\hline
\end{tabular}

Table 5. Masses of the real complex structure moduli $u_{1}, u_{2}$ the dilaton $s$ and their corresponding axion fields $\nu_{1}, \nu_{2}$ and $\sigma$. For the calculation of the masses the prefactor $\left\langle e^{K_{\text {Kähler }}}\right\rangle \simeq 3.7 \cdot 10^{-4}$ from the Kähler moduli stabilization, see table 4 , has been taken into account.

- The flux solution for $f$ and $h$ is inserted into

$$
0=\left(D_{S} W_{0}, D_{U_{1}} W_{0}, D_{U_{2}} W_{0}\right)_{(\text {inst. })}
$$

where this time the instanton corrections to the prepotential eq. (6.11) are taken into account in calculating $D_{i} W_{0}$ and the constant $\xi$ is set to its exact value. This generates shifts in the VEVs of $S, U_{1}$ and $U_{2}$ from their original rational values. Also the superpotential $W_{0}$ may be shifted from its zero value, eq. (6.15) to a nonvanishing value. If the resulting values for $S$ and $W_{0}$ are suitable for the Kähler moduli stabilization, we have constructed a de Sitter vacuum.

Let us present the solution that provides the parameters of our example given in eq. (6.5). The flux vector

$$
(f ; h)=(-16,0,0,0,-4,-2 ; 0,0,2,-8,-3,0),
$$

induces a D3 charge $Q_{D 3}^{f, h}=66$. Since this does not saturate the negative contribution to the total D3 tadpole, one has to switch on additional trivial gauge flux on the brane stacks or introduce a number of D3-branes to obtain an overall vanishing D3 charge.

The VEVs of the moduli and superpotential are

$$
\langle S\rangle=6.99, \quad\left\langle U_{1}\right\rangle=1.01, \quad\left\langle U_{2}\right\rangle=0.967, \quad\left\langle\left|W_{0}\right|\right\rangle=0.812 .
$$

A posteriori, we see that the chosen values for $A_{1}$ and $A_{2}$ in eq. (6.5) are indeed of the same order as the VEVs of the complex structure moduli. Furthermore, the assumption of working in the large complex structure limit is valid since the VEVs of $U_{1}$ and $U_{2}$ fulfill the condition that the instanton corrections are small, $U_{2} \gg 1 / 6$ and $U_{1}>1$ [70]. Finally, we calculate the masses of the complex structure and dilaton moduli to 0th order from the tree level potential $V=e^{K} K^{a \bar{b}} D_{a} W_{0} \overline{D_{b} W_{0}}$ for $a, b=U_{1}, U_{2}, S$ in table 5. A posteriori, we verify that there is indeed a separation of scales, i.e. the complex structure moduli and the dilaton are stabilized at a mass scale roughly two orders of magnitude higher than the Kähler moduli.

To conclude this section, we have explicitly constructed a de Sitter vacuum with all geometric moduli stabilized on $X_{3}$. The stabilization of the two Kähler moduli, the dilaton and two complex structure moduli has been carried out explicitly while the remaining 270 complex structure moduli are stabilized according to general arguments. 


\section{Conclusions}

We discussed the construction of explicit global models in a type IIB context, which exhibit the dynamics of Kähler uplifting. In this mechanism the interplay of gaugino condensation on 7-branes and the leading $\mathcal{O}\left(\alpha^{\prime 3}\right)$-correction fixes the Kähler moduli in a SUSY breaking minimum, after three-form flux has supersymmetrically stabilized the complex structure moduli and the axio-dilaton. The vacuum energy of this minimum can be dialed from AdS to dS by adjusting the flux-induced Gukov-Vafa-Witten superpotential using the flux discretuum. Both SUSY breaking and lifting to dS are driven by an F-term of the Kähler moduli sector arising from the presence of the $\alpha^{\prime}$-correction of the Kähler potential. Thus the dS uplift is realized entirely by the geometric closed string moduli. This was the motivation for trying to construct a fully explicit consistent global model including explicit flux choice and complex structure moduli stabilization.

In Kähler uplifted dS vacua the $\mathrm{CY}$ volume scales as $\mathcal{V} \propto N^{3 / 2}$ with $N$ the rank of the condensing gauge group living on the 7-brane stack wrapping the large four-cycle. Moreover, the scale of Kähler moduli stabilization and the resulting Kähler moduli masses are suppressed by an additional $\mathcal{O}(1 / \mathcal{V})$ compared to the scale of flux-induced complex structure moduli stabilization. Hence, we searched for a large gauge group rank to obtain a large volume.

We considered models which can be easily uplifted to F-theory compactifications on elliptically fibered CY fourfolds (embedded in toric spaces). We focused on models that possess Sen's weak coupling limit since we use the leading $\alpha^{\prime}$ correction which is not understood for generic points in the F-theory moduli space. Such models have the characteristic feature that the orientifold plane has to be in a homology class of high degree in order to obtain a singularity of large rank $N$.

We checked the consistency conditions to have a globally defined construction, e.g. that the toric base of the elliptically fibered fourfold should be free of singularities of any kind as well as the Calabi-Yau threefold hypersurface in the weak coupling limit. We found that in general this turns out to be a severe constraint when one tries to increase the class of the orientifold by choosing appropriately the weights defining the toric variety.

We then made sure that the volume was of swiss cheese type $\mathcal{V} \sim \mathcal{V}_{1}^{3 / 2}-\mathcal{V}_{i}^{3 / 2}$, or at least approximately swiss cheese, e.g $\mathcal{V} \sim\left(\mathcal{V}_{1}+\mathcal{V}_{i}\right)^{3 / 2}-\mathcal{V}_{i}^{3 / 2}$. Then one can manufacture a large overall volume by making $\mathcal{V}_{1}$ large by enforcing a large gauge group rank on the corresponding divisor $D_{1}$. We ensure that other large rank stacks wrapping some divisors $D_{i \neq 1}$ (possibly enforced by imposing large rank on $D_{1}$ ) did not destroy the large volume approximation.

Finally, we checked that the number of the neutral and charged zero modes could be put to zero, such that the gaugino condensation contribution to the superpotential is nonzero. To do this, the gauge flux on the brane stack has to be chosen appropriately: On the one hand it must be non-zero to 'rigidify' the wrapped divisor (if this is not rigid). On the other hand, it should be possible to tune the flux such that it does not generate additional zero modes in the form of chiral matter, charged under the condensing gauge group.

We studied constraints on large gauge group rank by discussing Kreuzer-Skarke models 
and hypersurfaces in toric varieties. For the subclass of threefolds with an elliptic F-theory lift $\left(\sim 10^{5}\right.$ models $)$ we extracted the distribution of the largest-rank gauge group as a function of the number of Kähler moduli $h^{1,1}$.

Choosing $\mathbb{C P}_{1169}^{4}[18]$, which has $h^{1,1}=2$ and $h^{2,1}=272$, as our explicit example we constructed large-rank singularities on a choice of two divisors, and analyzed the consistency constraints both in the type IIB Sen limit, and from the F-theory perspective. The emerging situation for $\mathbb{C P}_{11169}^{4}[18]$ looks summarily as follows: We construct an $\operatorname{Sp}(24)$ singularity on the 'large' divisor $D_{1}$, which is rigidified by gauge flux, breaking $\operatorname{Sp}(24)$ to $\mathrm{SU}(24)$. The presence of the $\mathrm{Sp}(24)$ stack forces an $\mathrm{SO}(24)$ singularity on the second divisor $D_{5}$, which already is rigid. The gauge flux can be tuned such that no further zero-modes are generated. In F-theory, the gaugino condensation superpotential is related to the superpotential generated by the M5-instantons wrapping the exceptional divisors $E_{i}$ in the resolved fourfold. In the considered case, we found that the exceptional divisors resolving the $\operatorname{Sp}(24)$ singularity satisfy $\chi_{0}\left(E_{i}\right) \geq 1$, that is the necessary condition in the presence of fluxes such that the wrapped M5-instantons contribute to the superpotential.

From the general results for supersymmetric flux stabilization we know, that at the no-scale level the resulting scalar potential is positive semi-definite, which yields full stability of the complex structure sector and the dilaton once fluxes fix them at an isolated supersymmetric point. As Kähler moduli stabilization via Kähler uplifting proceeds by breaking the no-scale structure at sub-leading order in the volume (like LVS), the stability of the flux-stabilized complex structure sector extends to the full model. We then analyzed the scalar potential that stabilizes the Kähler moduli. This singled out a band in $g_{s}-W_{0}$ plane where one finds de Sitter vacua. Here $W_{0}$ denotes the VEV of the GukovVafa-Witten superpotential arising from supersymmetric flux stabilization of the complex structure moduli. The overall volume of the Calabi-Yau threefold was determined by the data of the construction to be $\mathcal{V} \sim 52$.

The complex structure moduli space of $\mathbb{C P}_{11169}^{4}[18]$ possesses a high-order discrete symmetry $\Gamma$. We only considered three-form fluxes that respect this symmetry. As a consequence, all the $h^{2,1}-2$ non-invariant complex structure moduli are stabilized. The prepotential of the remaining two complex structure moduli is known via mirror symmetry, and this enables us to stabilize all $h^{2,1}$ moduli explicitly. It is this fact that in the end allowed us to construct a completely stabilized de Sitter vacuum.

Finally, we gave an explicit flux choice which stabilizes the axio-dilaton and the two $\Gamma$-invariant complex structure moduli at the right VEVs and value for $W_{0}$ for the Kähler stabilization of the explicit construction to proceed into a metastable dS vacuum. In summary, we gave a construction of an example for dS space in string theory which we believe to be explicit and complete within the limits of existing knowledge.

\section{Acknowledgments}

We thank Andreas Braun, Volker Braun, Michele Cicoli, Andres Collinucci, Frederik Denef, Iñaki García-Etxebarria, Thomas Grimm, James Halverson, Arthur Hebecker, Shamit Kachru, Magdalena Larfors, Christoph Mayrhofer, Danny Martínez Pedrera, Liam McAl- 
lister, Raffaele Savelli, Gary Shiu, Washington Taylor, Stefan Theisen, Timo Weigand, and Timm Wrase for valuable, and enlightening discussions, and useful comments. This work was supported by the Impuls und Vernetzungsfond of the Helmholtz Association of German Research Centers under grant HZ-NG-603, the German Science Foundation (DFG) within the Collaborative Research Center 676 "Particles, Strings and the Early Universe" and the Research Training Group 1670.

\section{A Models from two line weight systems}

In this appendix we want to discuss the constraints on building large gauge groups in Calabi-Yau threefolds that are complete intersections of hypersurfaces in a projective ambient space that is characterized by a weight system of two lines. This restriction is for simplicity. In all the approaches discussed below we find that going to arbitrarily high gauge groups would correspond to introducing singularities in the threefold of different kinds. In this sense, this appendix is meant as a summary of what can go wrong when one tries to build large gauge groups in a singularity free compact Calabi-Yau.

\section{A.1 One hypersurface Calabi-Yaus}

In this section we will analyze the threefold that is the hypersurface

$$
\xi^{2}-P_{2 n+6,4}=0
$$

in the ambient space

$$
X_{4}^{\mathrm{amb}}: \begin{array}{ccccccc}
u_{1} & u_{2} & u_{3} & u_{4} & u_{5} & \xi \\
\cline { 2 - 6 } & 1 & 1 & 1 & n & 0 & n+3 \\
& 0 & 0 & 0 & 1 & 1 & 2
\end{array} .
$$

Notice that in the case $n>6$ a $u_{5}^{2}$ term factors out from the polynomial $P_{2 n+6,4}$ due to the imposed scalings. The case $n=6$, that was studied in detail in section 3 , has the largest $n$ where the enforced factorization of $P_{2 n+6,4}$ is only linear in $u_{5}$. In the linear case, the O7 plane splits into two planes that for $n=6$ are not intersecting. When $n>6$ the picture is more complicated as there are intersecting $O 7$ planes, which produce orbifold singularities on the Calabi-Yau threefold.

Notice that the constraint on $n$ is less restrictive if one gives up the weak coupling limit and goes to a strongly coupled F-theory compactification. For example, when $n=18$ the following factorization on $u_{5}$ in enforced on the Tate polynomials $a_{i}$ :

$$
\begin{aligned}
& a_{1}=u_{5}^{1} A_{21,1}, \\
& a_{2}=u_{5}^{2} A_{42,2}, \\
& a_{3}=u_{5}^{3} A_{63,3}, \\
& a_{4}=u_{5}^{4} A_{84,4}, \\
& a_{6}=u_{5}^{5} A_{126,7},
\end{aligned}
$$

which corresponds to an $E_{8}$ singularity as one can look up in [58]. For $n>18$ there is a factorization $a_{6}=u_{5}^{m} A_{18+6 n, 12-m}$ with $m \geq 6$. This singularity cannot be resolved 
according to the Tate procedure and hence $n=18$ is the maximum value we can obtain in a strongly coupled F-theory setting. See also [69] for a discussion of this limit on $n$ derived in the Weierstrass parametrization of the fourfold.

Finally, note that the class of the orientifold is $[O 7]=21\left[D_{1}\right]+2\left[D_{5}\right]$ in the $n=18$ case while it is $[O 7]=9\left[D_{1}\right]+2\left[D_{5}\right]$ in the weakly coupled $n=6$ case. Hence, in principle one could construct a much larger gauge group on $D_{1}$ if one gives up the weak coupling limit. If the leading $\alpha^{\prime}$-correction of the Kähler potential were known in the strong coupling regime one could use this to construct Kähler uplifted de Sitter vacua where the large volume regime is more easily achieved.

\section{A.2 Complete intersections}

One can also study complete intersections in higher dimensional toric varieties. For instance, take a threefold that is defined by two equations in a five dimensional ambient toric variety. This corresponds to a base which is a hypersurface in a four-dimensional ambient toric variety [40]. In the remainder of this section, we demonstrate in an example that if one wants to have a non-singular base of the corresponding F-theory uplift and an approximately swiss-cheese intersection form, this constraints how large the coefficients in $\bar{K}$ and hence in $[O 7]$ can become. Also in other examples that we considered, trying to enlarge $\bar{K}$ at some point introduces singularities in the base or the double cover Calabi-Yau threefold.

As a working example, take the base manifold $B_{n l}$ that can be described as a hypersurface $P_{m, 2}\left(u_{i}\right)=0$ with positive integer $m$ in the projective space

$$
B_{n l}^{\mathrm{amb}}: \begin{array}{ccccccc}
u_{1} & u_{2} & u_{3} & u_{4} & u_{5} & u_{6} \\
\hline 1 & 1 & 1 & n & l & 0 \\
0 & 0 & 0 & 1 & 1 & 1
\end{array} .
$$

The ambient four complex dimensional toric variety eq. (A.4) can be interpreted as a $\mathbb{C P}^{2}$ fibration over a $\mathbb{C P}^{2}$ with integer twists $n$ and $l$ which we choose to be positive. Note that the degrees of $P$ have been chosen such that the anti-canonical bundle of $B_{n l}$ is $\bar{K}=(3+n+l-m)\left[D_{1}\right]+\left[D_{6}\right]$. In the previous section, the factorization of $u_{5}^{2}$ for $n>6$ in the $\xi^{2}-P_{2 n+6,4}=0$ equation in eq. (A.1) was due to the relation $\bar{K} \sim 2\left[D_{5}\right]$. We want to avoid this bound on $n$ here by making $\bar{K} \sim\left[D_{6}\right]$.

Factoring out those coordinates that are sections of the bundle $\mathcal{L}_{2}$ defined by the second line in eq. (A.4), the hypersurface equation is given as

$$
P_{m, 2}\left(u_{i}\right)=u_{6}^{2} P_{m}+u_{6} u_{5} P_{m-l}+u_{5}^{2} P_{m-2 l}+u_{6} u_{4} P_{m-n}+u_{5} u_{4} P_{m-l-n}+u_{4}^{2} P_{m-2 n},
$$

where the $P_{i}$ are sections only in the bundle $\mathcal{L}_{1}$ corresponding to the first line of eq. (A.4). To avoid factorization of eq. (A.5) in $u_{6}$ and hence a singularity in the base, we have to impose

$$
2 l \leq m .
$$

Note that the roles of $n$ and $l$ can be exchanged since they just correspond to a redefinition of the coordinates $u_{4}$ and $u_{5}$. If we wanted $l$ to obtain large values compared to $m$ and $n$ we should impose $2 n \leq m . \bar{K}$ obtains a large scaling in $\mathcal{L}_{1}$ as long as $n$ or $l$ are allowed to 
be large. In the following, we denote divisors in the ambient space corresponding to $u_{i}=0$ by $\tilde{D}_{i}$ while we symbolize the pullback on the base manifold by $D_{i}$.

Singularities of the ambient space. The fan of the toric variety $B_{n l}^{\mathrm{amb}}$ can be generated by the lattice vectors

$$
v_{1}=\left(\begin{array}{l}
-n \\
-l \\
-1 \\
-1
\end{array}\right), v_{2}=\left(\begin{array}{l}
0 \\
0 \\
1 \\
0
\end{array}\right), v_{3}=\left(\begin{array}{l}
0 \\
0 \\
0 \\
1
\end{array}\right), v_{4}=\left(\begin{array}{l}
1 \\
0 \\
0 \\
0
\end{array}\right), v_{5}=\left(\begin{array}{l}
0 \\
1 \\
0 \\
0
\end{array}\right), v_{6}=\left(\begin{array}{c}
-1 \\
-1 \\
0 \\
0
\end{array}\right)
$$

$B_{n l}^{\mathrm{amb}}$ is non-singular if all cones of the fan that generates the toric variety are generated by a subset of a lattice basis of the (in this case) four dimensional lattice. This is the case if all combinations $\operatorname{span}_{\mathbb{Z}}\left\{v_{i}, v_{j}, v_{k}, v_{l}\right\}$ for $i, j, k, l \in\{1, \ldots, 6\}$ that are part of the toric variety form a basis of the lattice space. For the fan spanned by the $v_{i}$ 's of eq. (A.7) the combinations that do not span the lattice are

$$
\begin{aligned}
& \left\{v_{1}, v_{2}, v_{3}, v_{4}\right\},\left\{v_{1}, v_{2}, v_{3}, v_{4}\right\},\left\{v_{1}, v_{2}, v_{3}, v_{4}\right\}, \\
& \left\{v_{1}, v_{4}, v_{5}, v_{6}\right\},\left\{v_{2}, v_{4}, v_{5}, v_{6}\right\},\left\{v_{3}, v_{4}, v_{5}, v_{6}\right\} .
\end{aligned}
$$

We will show in the next paragraph that $\tilde{D}_{1} \tilde{D}_{2} \tilde{D}_{3}$ and $\tilde{D}_{4} \tilde{D}_{5} \tilde{D}_{6}$ are part of the StanleyReisner ideal and hence the cones corresponding to eq. (A.8) do not belong to the toric variety which is thus free of singularities.

Intersections in the ambient space. We will now derive the quadruple intersections in the ambient space by analyzing the system of equations defined by the GLSM [52] of eq. (A.4)

$$
\begin{aligned}
\left|x_{1}\right|^{2}+\left|x_{2}\right|^{2}+\left|x_{3}\right|^{2}+n\left|x_{4}\right|^{2}+l\left|x_{5}\right|^{2}+\left|x_{6}\right|^{2} & =\xi_{1}>0 \\
\left|x_{4}\right|^{2}+\left|x_{5}\right|^{2}+\left|x_{6}\right|^{2} & =\xi_{2}>0 .
\end{aligned}
$$

A GLSM is a description of a toric variety where the D-flatness conditions of eq. (A.9) can be thought of as gauge fixing the absolute values of the rescalings in a complex projective space, while the $\mathrm{U}(1)$ symmetry associated to the phase of the rescalings remains to be divided out. We immediately see from eq. (A.9) that $\tilde{D}_{4} \tilde{D}_{5} \tilde{D}_{6}=0$. Consider now the intersections

$$
\begin{aligned}
& \tilde{D}_{1} \tilde{D}_{2} \tilde{D}_{3} \tilde{D}_{4}:\left|x_{5}\right|^{2}=\frac{\xi_{1}}{l},\left|x_{6}\right|^{2}=\frac{1}{l}\left(l \xi_{2}-\xi_{1}\right), \\
& \tilde{D}_{1} \tilde{D}_{2} \tilde{D}_{3} \tilde{D}_{5}:\left|x_{4}\right|^{2}=\frac{\xi_{1}}{n},\left|x_{6}\right|^{2}=\frac{1}{n}\left(n \xi_{2}-\xi_{1}\right), \\
& \tilde{D}_{1} \tilde{D}_{2} \tilde{D}_{3} \tilde{D}_{6}:\left|x_{4}\right|^{2}=\frac{1}{l-n}\left(l \xi_{2}-\xi_{1}\right),\left|x_{6}\right|^{2}=\frac{1}{l-n}\left(\xi_{1}-n \xi_{2}\right) .
\end{aligned}
$$

Independently of $n$ and $l$ these intersections vanish if

$$
\xi_{1}>n \xi_{2} \quad \text { and } \quad \xi_{1}>l \xi_{2} .
$$


In this case, $\tilde{D}_{1} \tilde{D}_{2} \tilde{D}_{3}$ and $\tilde{D}_{4} \tilde{D}_{5} \tilde{D}_{6}$ are elements of the Stanley Reisner ideal and hence the ambient space is singularity free. That the inequalities eq. (A.11) are indeed fullfilled will be shown in the next section.

To calculate all quadruple intersections it is sufficient to calculate the intersections for a basis of divisors, we pick $\tilde{D}_{1}$ and $\tilde{D}_{6}$. All other intersections can be calculated using the equivalences

$$
\tilde{D}_{1}=\tilde{D}_{2}=\tilde{D}_{3}, \tilde{D}_{4}=n \tilde{D}_{1}+\tilde{D}_{6}, \tilde{D}_{5}=l \tilde{D}_{1}+\tilde{D}_{6}
$$

Under the assumption eq. (A.11) we can show $\tilde{D}_{1}^{2} \tilde{D}_{5} \tilde{D}_{6}=1$. Successive use of the relations eq. (A.12) gives us the quadruple intersections

$$
\tilde{D}_{1}^{2} \tilde{D}_{6}^{2}=1, \quad \tilde{D}_{1} \tilde{D}_{6}^{3}=-n-l, \quad \tilde{D}_{6}^{3}=n^{2}+l(n+l) .
$$

Mori- and Kähler cone of the ambient space. The Mori cone, i.e. the set of twocycles that generate the full set of all two-cycle classes with holomorphic representatives, is spanned by $C_{1}=\tilde{D}_{1} \tilde{D}_{6}^{2}$ and $C_{2}=\tilde{D}_{1}^{2} \tilde{D}_{6}$. The weight matrix

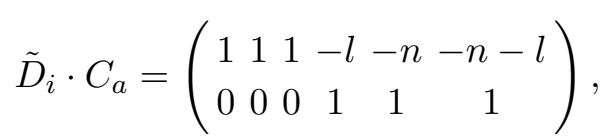

can be obtained from the original GLSM description eq. (A.4) by adding the second line of eq. (A.4) multiplied by $-n-l$ to the first line. This corresponds to the transformation of the Fayet-Iliopoulos terms of the GLSM according to

$$
\left(\xi_{1}, \xi_{2}\right) \rightarrow\left(\xi_{1}^{\prime}, \xi_{2}^{\prime}\right)=\left(\xi_{1}-(n+l) \xi_{2}, \xi_{2}\right)
$$

For a basis of divisors $K_{i}$ that is dual to the $C_{a}$, i.e. $K_{i} C_{a}=\delta_{i a}$ the Kähler form can be parametrized as $J=\xi_{i}^{\prime} K_{i}$. In this case, $K_{1}=\tilde{D}_{1}$ and $K_{2}=(n+l) \tilde{D}_{1}+\tilde{D}_{6}$. The Kähler cone, i.e. the space of all two forms with

$$
\int_{C_{i}} J>0
$$

for all $C_{i}$ in the Mori cone, is then simply given by

$$
\xi_{1}^{\prime}>0, \xi_{2}^{\prime}>0 \quad \Leftrightarrow \quad \xi_{1}-(n+l) \xi_{2}>0, \quad \xi_{2}>0 .
$$

The inequalities in eq. (A.17) a posteriori justify the quadruple intersections calculated in eq. (A.11).

Intersections in the base and volume form. The intersections of the base divisors $D_{i}$ are calculated from those in the ambient space via

$$
\kappa_{i j k}=\int_{B_{\mathrm{nl}}} D_{i} D_{j} D_{k}=\int_{B_{\mathrm{nl}}^{\mathrm{amb}}}\left(m \tilde{D}_{1}+2 \tilde{D}_{6}\right) \tilde{D}_{i} \tilde{D}_{j} \tilde{D}_{k}
$$


Using eq. (A.13) we thus find

$$
\begin{aligned}
D_{1}^{3} & =0 \\
D_{1}^{2} D_{6} & =2 \\
D_{1} D_{6}^{2} & =m-2(l+n), \\
D_{6}^{3} & =-m(l+n)+2\left(n^{2}+l(l+n)\right) .
\end{aligned}
$$

For the purpose of moduli stabilization the volume of the Calabi-Yau ${ }^{14}$

$$
\mathcal{V}=2 \mathcal{V}_{B_{\mathrm{nl}}}=\frac{1}{3} \int_{B_{\mathrm{nl}}} J^{3}=\frac{1}{3} \int_{B_{\mathrm{nl}}}\left(\xi_{1} D_{1}+\xi_{2} D_{6}\right)^{3}
$$

should be 'approximately swiss cheese', i.e. of the form

$$
\mathcal{V} \sim\left(a V_{D_{1}}+b V_{D_{6}}\right)^{3 / 2}-c V_{D_{6}}^{3 / 2} \quad \text { with } a, c>0 .
$$

As was discussed in [84], this can only be arranged if the two dimensional matrix

$$
A_{i j}=\kappa_{i j k} a^{k} \quad \text { for } \quad i, j, k=1,2,
$$

with triple intersections $\kappa_{i j k}$ and $a^{k} \in \mathbb{Z}$ defining the divisor $a^{1} D_{1}+a^{2} D_{6}$ can be brought into a special form: There is exactly one non-vanishing eigenvalue while the eigenvector corresponding to the vanishing eigenvalue is $(0,1)$. From the matrix entries

$$
\begin{aligned}
& A_{11}=2 a_{2}, \\
& A_{12}=A_{21}=2 a_{1}+a_{2}(m+2(-l-n)), \\
& A_{22}=a_{1}(m+2(-l-n))+a_{2}\left(m(-l-n)+2\left(n^{2}+l(l+n)\right)\right),
\end{aligned}
$$

we see that these conditions can only be met if

$$
m=2 l, a^{1}=n a^{2} \quad \text { or } \quad m=2 n, a^{1}=l a^{2} .
$$

Since we are interested in large $n$ having already imposed $2 l \leq m$ in eq. (A.6) we stick to the first option in eq. (A.24) and furthermore choose $a^{2}=1$. Now the volume can be shown to be given by

$$
\mathcal{V}=\frac{2}{3 n}\left[\left(n V_{D_{1}}+V_{D_{6}}\right)^{3 / 2}-V_{D_{6}}^{3 / 2}\right]
$$

The anti-canonical bundle of $B_{\mathrm{nl}}$ becomes

$$
\bar{K}=(3+n-l)\left[D_{1}\right]+\left[D_{6}\right] .
$$

Now going back to eq. (A.5) we see that the only way we can get a larger class than $3\left[D_{1}\right]$ in $\bar{K}$ is by demanding $u_{4}^{2} P_{m-2 n}=u_{4}^{2} P_{2(l-n)}=0$. This would however leave us with a singular base since along the curve $\left\{u_{5}=u_{6}=0\right\}$, which is not in the SR-ideal, we would have $P_{m, 2}=d P_{m, 2}=0$. Hence, we find the constraint $n-l \leq 0$ and we did not succeed in constructing a large class of $\left[D_{1}\right]$ in $\bar{K}$, as one can see from eq. (A.26).

\footnotetext{
${ }^{14}$ Note that the relation between the volume of the base and the volume of the Calabi-Yau could be more complicated as it was for instance the case for the threefold $X_{3}$ that we considered in section 3 .
} 

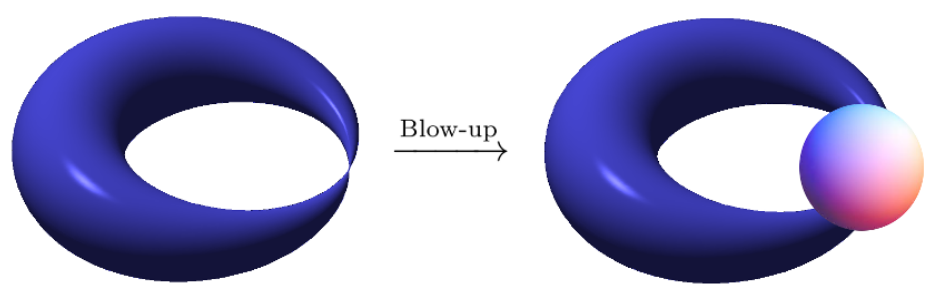

Figure 4. Visualization of a blown up $\operatorname{Sp}(1)$ singularity.

\section{B The $\operatorname{Sp}(N)$ resolved fourfold}

\section{B.1 Geometry of the $\operatorname{Sp}(N)$ resolved fourfold}

In this section, following the procedure used in [85, 86], we resolve the $\operatorname{Sp}(N)$ singularity on a divisor $\hat{D}$ for a general base. $\hat{D}$ is defined by an equation $p_{\hat{D}}=0$, where $p_{\hat{D}}$ is a polynomial of the base coordinates with proper degrees. ${ }^{15}$

The elliptic fibration becoming singular can be visualized by a pinching of the fibered torus, i.e. one of the torus cycles shrinks to zero size. The singularity is resolved via a series of blow-ups, where the blow-ups are such that they reproduce on the fiber the Dynkin diagram of the gauge group singularity that was originally imposed on the fiber. More precisely, the pullback to the base of the double intersections of the blown-up divisors build the Cartan matrix entries of the gauge group. For instance, the resolution of an $\mathrm{Sp}(1)$ singularity is visualized in figure 4 .

These blown-up divisors can also be formalized by means of toric geometry [85, 86]. To resolve an $\operatorname{Sp}(N)$ singularity, $N$ new coordinates $v_{2 i-1}, i=1, \ldots, N$, are introduced with projective scaling relations

\begin{tabular}{cc|cccccccc} 
& $\sigma$ & $X$ & $Y$ & $Z$ & $v_{1}$ & $v_{3}$ & $\cdots$ & $v_{2 N-3}$ & $v_{2 N-1}$ \\
\hline \multirow{2}{*}{$X_{6}^{\mathrm{amb}}$} & $:$ & 2 & 3 & 1 & 0 & 0 & $\cdots$ & 0 & 0 \\
& 1 & 1 & 1 & 0 & -1 & 0 & $\cdots$ & 0 & 0 \\
& $\vdots$ & 2 & 0 & 0 & -1 & $\cdots$ & 0 & 0 \\
& 1 & $\vdots$ & $\vdots$ & $\vdots$ & $\vdots$ & $\ddots$ & $\vdots$ & $\vdots$ \\
& 1 & $N-1$ & $N-1$ & 0 & 0 & 0 & $\cdots$ & -1 & 0 \\
& $N$ & $N$ & 0 & 0 & 0 & $\cdots$ & 0 & -1
\end{tabular}.

The resolved fourfold is embedded into the ambient sixfold $X_{6}^{\mathrm{amb}}$ (B.1) by the two equa-

\footnotetext{
${ }^{15}$ We will make no attempts to resolve the $\mathrm{SO}(N)$ singularity (for a discussion of an $\mathrm{SO}(10)$ resolution, see [87].) From the point of view of gaugino condensation in our example the resolution is not necessary since we know that $D_{5}^{\text {fix }}$ is rigid in the type IIB Calabi-Yau threefold, table 3. Resolving the $\mathrm{SO}(N)$ singularity would be necessary if we wanted to calculate the D3 tadpole from the F-theory perspective, as we would require the Euler number of the completely resolved fourfold.
} 
tions:

$$
Y^{\mathrm{res}}:\left\{\begin{aligned}
& Y\left(Y+a_{1} X Z+a_{3, N} \Pi_{i=0}^{N} v_{2 i-1}^{N-i} Z^{3}\right) \\
&=X^{3} \Pi_{i=0}^{N} v_{2 i-1}^{i}+a_{2} X^{2} Z^{2}+a_{4, N} \Pi_{i=0}^{N} v_{2 i-1}^{N-i} X Z^{4}+a_{6,2 N}\left(\Pi_{i=0}^{N} v_{2 i-1}^{N-i}\right)^{2} Z^{6}, \\
& p_{\hat{D}}=\Pi_{i=0}^{N} v_{2 i-1} .
\end{aligned}\right.
$$

where we have defined $v_{-1} \equiv \sigma$.

The vanishing of the new coordinates defines the exceptional divisors $E_{2 i-1}:\left\{v_{2 i-1}=\right.$ $0\}$ and $E_{-1} \equiv[\sigma]$. As far as divisor classes are concerned there are the following equivalences:

$$
[\sigma]=\hat{D}-\sum_{i=1}^{N} E_{2 i-1}, \quad[X]=2([Z]+\bar{K})-\sum_{i=1}^{N} i E_{2 i-1}, \quad[Y]=3([Z]+\bar{K})-\sum_{i=1}^{N} i E_{2 i-1}
$$

where $[X],[Y],[Z]$ are the homology classes of the divisors $\{X=0\},\{Y=0\},\{Z=0\}$. With abuse of notation we call $\hat{D}$ both the four-cycle $\left\{p_{\hat{D}}=0\right\}$ on the base and its uplift to the Calabi-Yau fourfold, i.e. a six-cycle that is an elliptic fibration over the locus $\left\{p_{\hat{D}}=0\right\}$ on the base.

\section{B.2 Intersections of exceptional divisors from the Stanley-Reisner ideal}

The Stanley-Reisner (SR) ideal of the fourfold with a resolved $\operatorname{Sp}(N)$ singularity is

$$
S R_{\mathrm{Sp}(N)}=\left\{X Y Z, v_{2 i-1} Z_{\mid i=1, \ldots, N}, v_{2 i-1} X_{\mid i=0, \ldots, N-1}, v_{2 i-1} v_{2 j-1 \mid i, j=0, \ldots, N ; j-i>1}\right\} .
$$

This follows from the SR ideal of the ambient sixfold given in [86], restricted to the fourfold. One can directly deduce eq. (B.4) from the fact that the SR ideal is the union of all sets

$$
Z_{I}=\left\{\left(z_{1}, \ldots, z_{n}\right) \mid z_{j}=0 \forall j \in I\right\}
$$

for which there is no cone, such that all the one-cones $\rho_{j}$ associated to the homogeneous coordinate $z_{j}$ with $j \in I$ lie in one cone. Let us consider the case of $\operatorname{Sp}(2)$ from where it is straightforward to deduce eq. (B.4). The SR ideal reads

$$
S R_{\mathrm{Sp}(2)}=\left\{X Y Z, v_{1} Z, v_{3} Z, \sigma X, v_{1} X, \sigma v_{3}\right\}
$$

This can be obtained via the following polytope construction: From the scaling relations eq. (B.1) one can make a canonical choice of one-cones $\rho_{i}[88,89]$ for the coordinate set $\left\{X, Y, Z, v_{2 i-1 \mid i=0, \ldots, N}\right\}$ :

$$
x=\left(\begin{array}{c}
-1 \\
0 \\
\underline{0}
\end{array}\right), y=\left(\begin{array}{c}
0 \\
-1 \\
\underline{0}
\end{array}\right), z=\left(\begin{array}{l}
2 \\
3 \\
\underline{0}
\end{array}\right), v_{2 i-1}=\left(\begin{array}{c}
2-i \\
3-i \\
\underline{v}
\end{array}\right),
$$

for $i=0, \ldots, N$. 


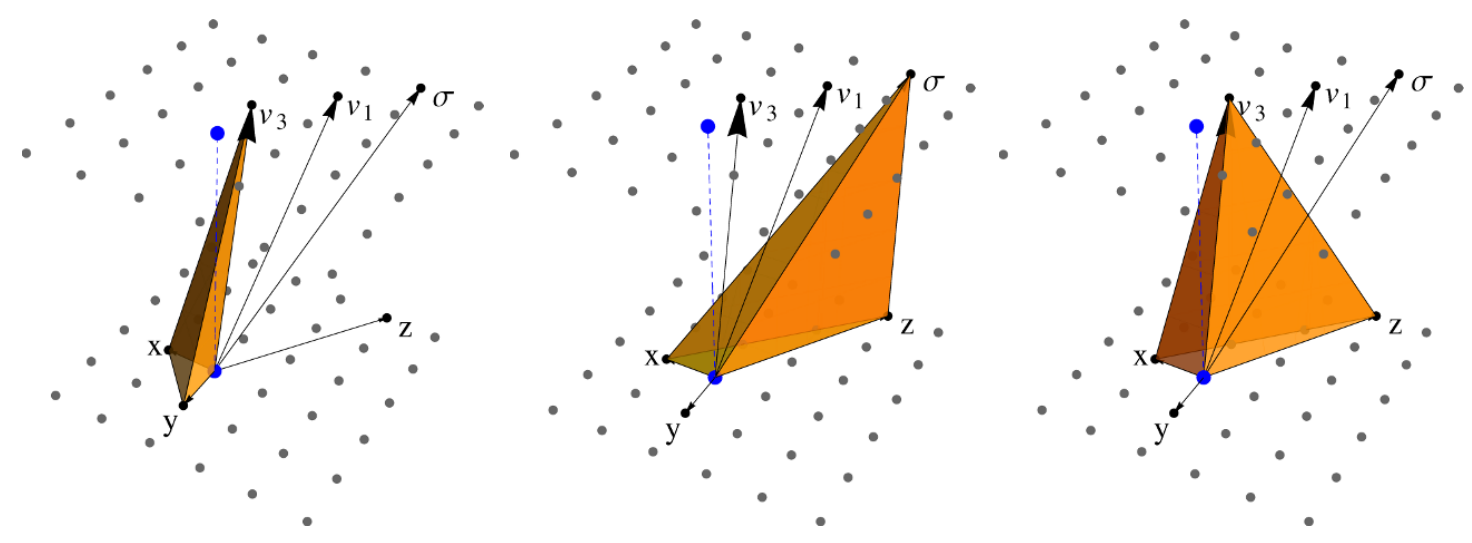

Figure 5. 3D projection of the fan of the $\mathrm{Sp}(2)$ resolution manifold for the subset of coordinates $\left\{X, Y, Z, \sigma, v_{1}, v_{3}\right\}$. The top layer of grey lattice points corresponds to the projection $\underline{v} \rightarrow 1$ while the bottom layer to $\underline{0} \rightarrow 0$. The blue point indicates the origin. In the first plot from the l.h.s., we see the cone spanned by $\left\{x, y, v_{3}\right\}$ is such that the one-cones $x, y$ and $z$ can never lie in one cone and hence $X Y Z$ is an element of the SR ideal. In the second plot, we see that the cone spanned by $\{x, z, \sigma\}$ is such that $z, v_{1}$ and $z, v_{3}$ respectively can never lie in one cone. In the third plot we see that the cone spanned by $\left\{x, z, v_{3}\right\}$ forces $X \sigma$ and $X v_{1}$ to lie in the SR ideal. $\sigma v_{3}$ is an element of the SR ideal because $v_{1}$ lies on a line that connects them and hence they can never lie in one cone. These are all possible elements of the SR ideal, notice that for example $y$ and $v_{1}$ lie in one cone: $\left\{y, v_{1}, v_{3}\right\}$.

Mapping $\underline{0} \rightarrow 0$ and $\underline{v} \rightarrow 1$ we can draw a three dimensional projection of the fan which we show in figure 5 for the case of $\operatorname{Sp}(2)$. Using the definition of the SR ideal one can read of its elements. For a more detailed explanation see the caption of figure 5 .

We now want to calculate the double intersections of the exceptional divisors $E_{2 i-1} E_{2 j-1}$ from the SR ideal (B.4) using in principal the same strategy as was presented in [61] to obtain the double intersections of the exceptional divisors for the $\mathrm{SU}(N)$-resolution manifold for $N=2, \ldots, 5$. In the $\operatorname{Sp}(N)$ case we consider here this analysis is more straightforward than in the $\mathrm{SU}(N)$ case and can actually be used to obtain the intersections for an arbitrary $\operatorname{Sp}(N)$ resolution manifold. This, in turn will be used to derive in the next section a formula for the arithmetic genus for the $\operatorname{Sp}(N)$ resolution manifold with arbitrary $N$.

The most general form of the double intersections is

$$
E_{2 i-1} E_{2 j-1}=C_{i j} \hat{D}([Z]+\bar{K})+d_{m} E_{2 m-1} \hat{D}+k_{m} E_{2 m-1} \bar{K}
$$

where $\hat{D}$ is the base divisor $\left\{p_{\hat{D}}=0\right\}$ where the $\operatorname{Sp}(N)$ singularity is located. The $C_{i j}$ have to be the entries of the Cartan matrix of $\operatorname{Sp}(N)$ times two, since $\operatorname{Sp}(N)$ is not simply-laced. The coefficients $d_{m}$ and $k_{m}$ can be extracted from the SR ideal and the relations (B.3). For example $[\sigma][X]=0$ implies:

$$
\left(\hat{D}-\sum_{k=1}^{N} E_{2 k-1}\right)\left(2([Z]+\bar{K})-\sum_{i=1}^{N} i E_{2 i-1}\right)=0 .
$$


In general, we have to solve $2 N-1$ linear equations

$$
\begin{aligned}
E_{2 i-1}[X]=0 & \text { for } i=0, \ldots, N-1, \\
{[\sigma] E_{2 i-1}=0 } & \text { for } i=2, \ldots, N .
\end{aligned}
$$

for $2 N-1$ non-zero intersections $E_{2 i-1 \mid i=1, \ldots, N}^{2}, E_{2 i-1} E_{2 i+1 \mid i=1, \ldots, N-1}$. These equations read

$$
\begin{aligned}
\sum_{j=2}^{N-1} j E_{2 j-1}\left(E_{2 j-3}+E_{2 j-1}\right. & \left.+E_{2 j+1}\right)+N E_{2 N-1}\left(E_{2 N-3}+E_{2 N-1}\right) \\
+E_{1}\left(E_{1}+E_{3}\right) & =-2 \hat{D}([Z]+\bar{K})+\hat{D} \sum_{j=2}^{N-1} j E_{2 j-1}+2 \bar{K} \sum_{j=2}^{N-1} E_{2 j-1}, \\
E_{1}\left(E_{1}+2 E_{3}\right) & =2 E_{1} \bar{K}, \\
E_{2 i-1}\left[(i-1) E_{2 i-3}+i E_{2 i-1}+(i+1) E_{2 i+1}\right] & =2 E_{2 i-1} \bar{K} ; \quad i=2, \ldots, N-1, \\
E_{2 i-1}\left[\hat{D}-E_{2 i-3}-E_{2 i-1}-E_{2 i+1}\right] & =0 ; \\
E_{2 N-1}\left[\hat{D}-E_{2 N-3}-E_{2 N-1}\right] & =0 .
\end{aligned}
$$

It can be shown by mathematical induction $\forall N$ that these linear equations are solved by the following non-zero double intersections:

$$
\begin{aligned}
E_{2 i-1} E_{2 i+1}= & 2 \hat{D}([Z]+\bar{K})-2 \bar{K} \sum_{k=i+1}^{N} E_{2 k-1}-\hat{D} \sum_{k=1}^{i} k E_{2 k-1} ; \quad i=1, \ldots, N-1, \\
E_{2 i-1}^{2}= & -4 \hat{D}([Z]+\bar{K})+\bar{K}\left(4 \sum_{k=i+1}^{N} E_{2 k-1}+2 E_{2 i-1}\right) \\
& +\hat{D}\left(2 \sum_{k=i+1}^{i-1} k E_{2 k-1}+(i+1) E_{2 i-1}\right) ; \quad i=1, \ldots, N-1, \\
E_{2 N-1}^{2}= & -2 \hat{D}([Z]+\bar{K})+2 \bar{K} E_{2 N-1}+\hat{D}\left(\sum_{k=1}^{N-1} k E_{2 k-1}+E_{2 N-1}\right) .
\end{aligned}
$$

\section{B.3 Arithmetic genus of divisors}

The arithmetic genus $\chi_{0}$ of a divisor $D$ in a fourfold $X_{4}^{\text {res }}$ is given by

$$
\begin{aligned}
\chi_{0}(D) & =\frac{1}{24} \int_{D} c_{1}(D) c_{2}(D) \\
& =\frac{1}{24} \int_{X_{4}^{\text {res }}}\left(-D^{2}\right)\left(c_{2}\left(X_{4}^{\mathrm{res}}\right)+D^{2}\right) .
\end{aligned}
$$


To calculate the second Chern class $c_{2}\left(X_{4}^{\text {res }}\right)$ over a base $B_{3}$ with toric divisors $\hat{D}_{i}$ note that the total Chern class is given by

$$
\begin{aligned}
c\left(X_{4}^{\text {res }}\right) & =c_{\text {fib. }} \prod_{\hat{D}_{i} \neq \hat{D}}\left(1+\hat{D}_{i}\right)(1+[\sigma])\left(1+E_{1}\right) \ldots\left(1+E_{2 N-1}\right) \\
& =c_{\text {fib. }} \prod_{\hat{D}_{i} \neq \hat{D}}\left(1+\hat{D}_{i}\right)\left(1+\sum_{j=0}^{N} E_{2 i-1}+\sum_{0 \leq k<l}^{N} E_{2 k-1} E_{2 l-1}\right) \\
& =c_{\text {fib. }}\left(1+\bar{K}+c_{2}(B)+\hat{D} \sum_{j=1}^{N} E_{2 i-1}-\sum_{1 \leq k<l}^{N} E_{2 k-1} E_{2 l-1}+\ldots\right),
\end{aligned}
$$

where the dots denote terms that are at least triple intersections and

$$
c_{\text {fib. }}=\frac{(1+[X])(1+[Y])(1+[Z])}{1+2[Y]} .
$$

Using the relations (B.3) and the intersection formula eq. (B.12) it is then straightforward to show

$$
c\left(X_{4}^{\mathrm{res}}\right)=1+c_{2}(B)+12 \bar{K}[Z]+11 \bar{K}^{2}-7 \bar{K} \sum_{i=1}^{N} i E_{2 i-1}+\hat{D} \sum_{i=1}^{N} i^{2} E_{2 i-1}+\ldots,
$$

where the dots denote the third and higher Chern classes. The arithmetic genus of various divisors can then be calculated from the definition eq. (B.13) using again the intersection formula eq. (B.12) and $E_{2 i-1}[Z]=0_{\mid i=1, \ldots, N}$. We list the arithmetic genus of the divisors that are of interest for us:

$$
\begin{aligned}
\chi_{0}\left(E_{2 i-1}\right) & =\frac{1}{6} \int_{B_{3}} \hat{D}\left[c_{2}(B)+\bar{K}^{2}+2 \hat{D}^{2}\right], i=1, \ldots, N-1, \\
\chi_{0}\left(E_{2 N-1}\right) & =\frac{1}{12} \int_{B_{3}} \hat{D}\left[c_{2}(B)+\bar{K}^{2}-3 \bar{K} \hat{D}+2 \hat{D}^{2}\right] .
\end{aligned}
$$

One can check that

$$
\chi_{0}\left(E_{2 N-1}\right)=\chi_{0}(\hat{D}) \quad \text { and } \quad \chi_{0}\left(E_{2 i-1}\right)=\chi_{0}(D), \quad i=1, \ldots, N-1,
$$

where $\hat{D}$ is the divisor $\left\{p_{\hat{D}}=0\right\}$ on the base manifold $B_{3}$ and $D$ is its double cover in $X_{3}$. The relations (B.18) can be expected, by considering that the exceptional divisors $E_{2 i-1}$ are $\mathbb{P}^{1}$ fibrations over a divisor on the base manifold. ${ }^{16}$

\footnotetext{
${ }^{16}$ In particular, the elliptic fiber splits on top of $\left\{p_{\hat{D}}=0\right\}$ into a collection of $\mathbb{P}^{1}$ s whose mutual intersection is encoded into the Dynkin diagram of the corresponding ADE-singularity. When the singularity is 'nonsplit', i.e. some of the $\mathbb{P}^{1} \mathrm{~s}$ are interchanged by a monodromy when going around the base divisor, then the gauge group is not of A-D-E type. The monodromies are reflected by symmetries of the ADE Dynkin diagram, leading to the diagram of the B-C Lie groups. This is the case of the $\operatorname{Sp}(N)$ groups: For example, the Dynkin diagram of $\mathrm{Sp}(3)$ is given by mirroring the Dynkin diagram of $\mathrm{SU}(6)$ as shown in figure 6 in appendix C. Translated to the fourfold, this means that the $\mathbb{P}^{1}$ associated with the dashed node is trivial under the monodromies corresponding to the mirroring. The corresponding fourfold exceptional divisor $E_{2 N-1}$ is a $\mathbb{P}^{1}$ fibration over $\hat{D}$. On the other hand the other exceptional divisors $E_{2 i-1}(i=1, \ldots, N-1)$ are fibrations over $\hat{D}$ of two $\mathbb{P}^{1}$ s, exchanged under the monodromy, once one goes around $\hat{D}$. These cycles can also be seen as a $\mathbb{P}^{1}$ fibration over the double cover $D$ of $\hat{D}$. Using the fact that the arithmetic genus of a $\mathbb{P}^{1}$ fibration over a manifold $M$ is the same as the arithmetic genus of $M$, one can derive the relations (B.18).
} 


\section{Geometry of the SU(N) resolved fourfold}

In section B.1, we discussed the features of establishing and resolving an $\operatorname{Sp}(N)$ singularity. As it is known from the type IIB picture an $\operatorname{Sp}(N)$ gauge group is broken to an $\mathrm{SU}(N) \times \mathrm{U}(1)$ gauge group by switching on a suitable gauge flux on the brane stack. So we could also directly impose an $\mathrm{SU}(N)$ singularity in the F-theory picture even though there is an important difference to the breaking by flux that becomes apparent in the weak coupling limit.

Comparing the $\mathrm{SU}(2 N)$ and $\mathrm{Sp}(N)$ Tate factorization in table 1 there is an additional factorization of $a_{2}$ which has a drastic consequence in the Sen limit. The Calabi-Yau hypersurface equation (2.22) enforces a conifold singularity:

$$
0=\xi^{2}-\left(a_{1}^{2}+w_{i} a_{2,1}\right) \equiv x_{1} x_{2}-x_{3} x_{4} .
$$

where on the r.h.s. of eq. (C.1) we have used one of the standard parametrizations of the conifold [90], i.e. $x_{1}=\xi-a_{1}, x_{2}=\xi+a_{1}, x_{3}=w_{i}$ and $x_{4}=a_{2,1}$. Notice that this does not happen in the $\operatorname{Sp}(N)$ case since $a_{1}^{2}+a_{2}=0$ cannot be brought into the form that parametrizes a conifold. Note that the appearance of this singularity does not depend on the gauge group rank, as it appears for all $\mathrm{SU}(2 N)$. Since it is not known how to resolve this conifold singularity there is no smooth transition between the general F-theory picture and the perturbative type IIB picture $[59,61,86]$. In particular, we do not know if we can use the leading $\alpha^{\prime}$ correction to the Kähler potential of [60] which was derived in the smooth perturbative IIB picture.

The conifold sits at the point

$$
\xi=a_{1}=w_{i}=a_{2,1}=0 .
$$

We can check if this intersections exists in our example of an elliptically fibered fourfold over $B_{3}$ with an $\mathrm{SU}(N)$ gauge group enforced over $\hat{D}_{1}$

$$
\int_{B}\left[9 \hat{D}_{1}+2 \hat{D}_{5}\right] \wedge \hat{D}_{1} \wedge\left[18 \hat{D}_{1}+4 \hat{D}_{5}-\hat{D}_{1}\right]=22 .
$$

Hence, for our example we cannot avoid the conifold singularities.

Ignoring the problematics of the Sen limit of $\mathrm{SU}(N)$ gauge groups for the moment, we have attempted to calculate the arithmetic genus of the exceptional divisors in the resolved fourfold of an $\mathrm{SU}(N)$ singularity imposed on a divisor $\hat{D}$. The blow-up procedure to obtain the resolved fourfold which is nicely discussed in [61] for $\mathrm{SU}(2), \mathrm{SU}(3), \ldots, \mathrm{SU}(5)$ is more complicated than in the $\operatorname{Sp}(N)$ case due to the following features:

- The scaling relations of the blown-up $\mathbb{P}_{1}$ s labeled by exceptional divisors $e_{i}$ are e.g. for $\mathrm{SU}(4)$ given by

\begin{tabular}{c|cccccc}
$e_{0}$ & $X$ & $Y$ & $Z$ & $e_{1}$ & $e_{2}$ & $e_{3}$ \\
\hline 0 & 2 & 3 & 1 & 0 & 0 & 0 \\
1 & 1 & 1 & 0 & -1 & 0 & 0 \\
1 & 1 & 2 & 0 & 0 & 0 & -1 \\
1 & 2 & 2 & 0 & 0 & -1 & 0
\end{tabular}.



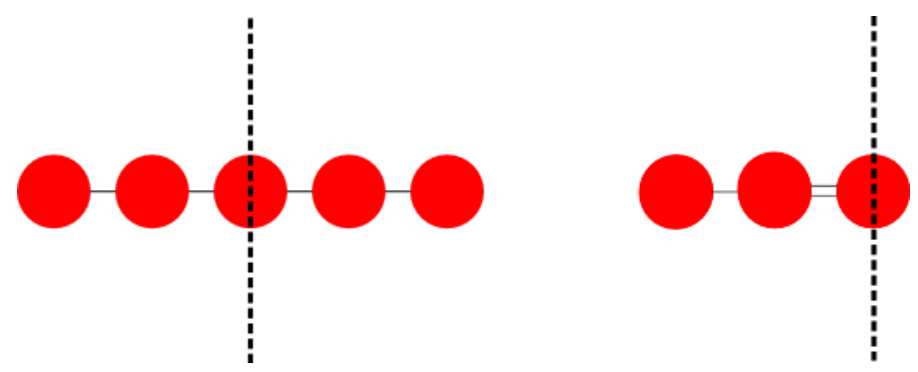

Figure 6. Dynkin diagrams for $\mathrm{SU}(6)$ (left) and $\mathrm{Sp}(3)$ (right). The $S p$ diagram can be obtained from the $S U$ diagram by mirroring the $S U$ diagram with respect to the dashed line. Only the blob which lies on the dashed line is invariant under this procedure.

There is a 'non-linear' scaling in $X$ and $Y$, i.e. the weights of $X$ and $Y$ of a line of weights are not proportional to the weights of the previous line which was the case in the $\operatorname{Sp}(N)$ case eq. (B.1). As a consequence the Stanley Reisner ideal is not as easily derived as eq. (B.4).

- The order in which the $e_{i}$ 's are introduced does not always reproduce the Cartan matrix of $\mathrm{SU}(N)$ in the double intersections of these exceptional divisors. This makes some relabeling inevitable which is reflected in the $\mathrm{SU}(4)$ example eq. (C.4) by the $e_{1}, e_{2}, e_{3}$ columns not containing a diagonal matrix with entries -1 .

However, the resolved fourfold including the double intersections of the $e_{i}$ were derived in [61] up to $\mathrm{SU}(5)$. We use the results of [61] to calculate the arithmetic genus of the exceptional divisors and find

$$
\chi_{0}\left(e_{i}\right)=\frac{1}{12} \int_{B} \hat{D} \wedge\left[c_{2}(B)+(\bar{K}-\hat{D}) \wedge(\bar{K}-2 \hat{D})\right] \quad \forall i<N \leq 5
$$

This result is the same as the $\operatorname{Sp}(N)$ case only for the last introduced exceptional divisor $E_{2 N-1}$, see eq. (B.17) while the divisors $E_{2 i-1}$ for $i<N$ obeyed a different formula. This is plausible as can be seen from the Dynkin diagrams of $\operatorname{Sp}(N)$ and $\mathrm{SU}(2 N)$, see figure 6 .

Using eq. (C.5) for our base $B$ and $\hat{D}=\hat{D}_{1}$ we see that all exceptional divisors have $\chi_{0}=1$ and hence could carry a non-perturbative superpotential from gaugino condensation. Even though we can only prove this for $\mathrm{SU}(N)$ with $N \leq 5$ we suspect that this result will hold for arbitrary $\mathrm{SU}(N)$ since the double intersections of the exceptional divisors are governed by the Cartan matrix which implies in particular that there is only an intersection of $e_{i}$ with the direct neighbors $e_{i-1}, e_{i}$ and $e_{i+1}$. Hence, going to larger gauge group the arithmetic genus of say $e_{1}$ should not be affected by the newly added divisors $e_{N}, e_{N-1}, \ldots$

Open Access. This article is distributed under the terms of the Creative Commons Attribution License which permits any use, distribution and reproduction in any medium, provided the original author(s) and source are credited. 


\section{References}

[1] M. Graña, Flux compactifications in string theory: a comprehensive review, Phys. Rept. 423 (2006) 91 [hep-th/0509003] [INSPIRE].

[2] M.R. Douglas and S. Kachru, Flux compactification, Rev. Mod. Phys. 79 (2007) 733 [hep-th/0610102] [INSPIRE].

[3] R. Blumenhagen, B. Körs, D. Lüst and S. Stieberger, Four-dimensional string compactifications with D-branes, orientifolds and fluxes, Phys. Rept. 445 (2007) 1 [hep-th/0610327] [INSPIRE].

[4] L. McAllister and E. Silverstein, String cosmology: a review, Gen. Rel. Grav. 40 (2008) 565 [arXiv:0710.2951] [INSPIRE].

[5] S.B. Giddings, S. Kachru and J. Polchinski, Hierarchies from fluxes in string compactifications, Phys. Rev. D 66 (2002) 106006 [hep-th/0105097] [InSPIRE].

[6] K. Dasgupta, G. Rajesh and S. Sethi, M theory, orientifolds and G-flux, JHEP 08 (1999) 023 [hep-th/9908088] [INSPIRE]

[7] S. Kachru, R. Kallosh, A.D. Linde and S.P. Trivedi, de Sitter vacua in string theory, Phys. Rev. D 68 (2003) 046005 [hep-th/0301240] [INSPIRE].

[8] V. Balasubramanian, P. Berglund, J.P. Conlon and F. Quevedo, Systematics of moduli stabilisation in Calabi-Yau flux compactifications, JHEP 03 (2005) 007 [hep-th/0502058] [INSPIRE].

[9] E. Silverstein, Simple de Sitter solutions, Phys. Rev. D 77 (2008) 106006 [arXiv:0712.1196] [INSPIRE].

[10] C. Caviezel et al., The Effective theory of type IIA AdS $S_{4}$ compactifications on nilmanifolds and cosets, Class. Quant. Grav. 26 (2009) 025014 [arXiv:0806.3458] [INSPIRE].

[11] S.S. Haque, G. Shiu, B. Underwood and T. Van Riet, Minimal simple de Sitter solutions, Phys. Rev. D 79 (2009) 086005 [arXiv:0810.5328] [INSPIRE].

[12] C. Caviezel et al., On the cosmology of Type IIA compactifications on $\mathrm{SU}(3)$-structure manifolds, JHEP 04 (2009) 010 [arXiv:0812.3551] [INSPIRE].

[13] R. Flauger, S. Paban, D. Robbins and T. Wrase, Searching for slow-roll moduli inflation in massive type IIA supergravity with metric fluxes, Phys. Rev. D 79 (2009) 086011 [arXiv:0812.3886] [INSPIRE].

[14] J. Polchinski and E. Silverstein, Dual purpose landscaping tools: small extra dimensions in AdS/CFT, arXiv:0908.0756 [INSPIRE].

[15] X. Dong, B. Horn, E. Silverstein and G. Torroba, Micromanaging de Sitter holography, Class. Quant. Grav. 27 (2010) 245020 [arXiv: 1005.5403] [INSPIRE].

[16] X. Dong, B. Horn, S. Matsuura, E. Silverstein and G. Torroba, FRW solutions and holography from uplifted AdS/CFT, Phys. Rev. D 85 (2012) 104035 [arXiv:1108.5732] [INSPIRE].

[17] V. Balasubramanian and P. Berglund, Stringy corrections to Kähler potentials, SUSY breaking and the cosmological constant problem, JHEP 11 (2004) 085 [hep-th/0408054] [INSPIRE]. 
[18] C. Burgess, R. Kallosh and F. Quevedo, de Sitter string vacua from supersymmetric D terms, JHEP 10 (2003) 056 [hep-th/0309187] [INSPIRE].

[19] K.A. Intriligator, N. Seiberg and D. Shih, Dynamical SUSY breaking in meta-stable vacua, JHEP 04 (2006) 021 [hep-th/0602239] [INSPIRE].

[20] O. Lebedev, H.P. Nilles and M. Ratz, de Sitter vacua from matter superpotentials, Phys. Lett. B 636 (2006) 126 [hep-th/0603047] [INSPIRE].

[21] M. Haack, D. Krefl, D. Lüst, A. Van Proeyen and M. Zagermann, Gaugino condensates and D-terms from D7-branes, JHEP 01 (2007) 078 [hep-th/0609211] [INSPIRE].

[22] D. Cremades, M.-P. Garcia del Moral, F. Quevedo and K. Suruliz, Moduli stabilisation and de Sitter string vacua from magnetised D7 branes, JHEP 05 (2007) 100 [hep-th/0701154] [INSPIRE].

[23] S. Krippendorf and F. Quevedo, Metastable SUSY Breaking, de Sitter Moduli Stabilisation and Kähler Moduli Inflation, JHEP 11 (2009) 039 [arXiv:0901.0683] [INSPIRE].

[24] M. Rummel and A. Westphal, A sufficient condition for de Sitter vacua in type IIB string theory, JHEP 01 (2012) 020 [arXiv:1107.2115] [INSPIRE].

[25] M. Cicoli, A. Maharana, F. Quevedo and C. Burgess, de Sitter string vacua from dilaton-dependent non-perturbative effects, JHEP 06 (2012) 011 [arXiv:1203.1750] [INSPIRE].

[26] M. Cicoli, S. Krippendorf, C. Mayrhofer, F. Quevedo and R. Valandro, D-Branes at del Pezzo singularities: global embedding and moduli stabilisation, JHEP 09 (2012) 019 [arXiv: 1206.5237] [INSPIRE].

[27] R. Bousso and J. Polchinski, Quantization of four form fluxes and dynamical neutralization of the cosmological constant, JHEP 06 (2000) 006 [hep-th/0004134] [INSPIRE].

[28] J.L. Feng, J. March-Russell, S. Sethi and F. Wilczek, Saltatory relaxation of the cosmological constant, Nucl. Phys. B 602 (2001) 307 [hep-th/0005276] [INSPIRE].

[29] F. Denef and M.R. Douglas, Distributions of flux vacua, JHEP 05 (2004) 072 [hep-th/0404116] [INSPIRE].

[30] D. Marsh, L. McAllister and T. Wrase, The Wasteland of random supergravities, JHEP 03 (2012) 102 [arXiv:1112.3034] [INSPIRE].

[31] X. Chen, G. Shiu, Y. Sumitomo and S.H. Tye, A global view on the search for de-Sitter vacua in (type IIA) string theory, JHEP 04 (2012) 026 [arXiv:1112.3338] [INSPIRE].

[32] T.C. Bachlechner, D. Marsh, L. McAllister and T. Wrase, Supersymmetric vacua in random supergravity, arXiv:1207.2763 [INSPIRE].

[33] Y. Sumitomo and S.-H.H. Tye, A stringy mechanism for a small cosmological constant, JCAP 08 (2012) 032 [arXiv: 1204.5177] [INSPIRE].

[34] Y. Sumitomo and S.-H.H. Tye, A Stringy Mechanism for A Small Cosmological Constant, in The 3rd UTQuest workshop ExDiP 2012 Superstring Cosmophysics, Obihiro Japan (2012).

[35] C. Vafa, Evidence for F-theory, Nucl. Phys. B 469 (1996) 403 [hep-th/9602022] [INSPIRE].

[36] A. Sen, Orientifold limit of F-theory vacua, Phys. Rev. D 55 (1997) 7345 [hep-th/9702165] [INSPIRE]. 
[37] M. Cicoli, C. Mayrhofer and R. Valandro, Moduli Stabilisation for Chiral Global Models, JHEP 02 (2012) 062 [arXiv:1110.3333] [INSPIRE].

[38] A. Westphal, de Sitter string vacua from Kähler uplifting, JHEP 03 (2007) 102 [hep-th/0611332] [INSPIRE].

[39] A. Collinucci, New F-theory lifts, JHEP 08 (2009) 076 [arXiv:0812.0175] [INSPIRE].

[40] A. Collinucci, New F-theory lifts. II. Permutation orientifolds and enhanced singularities, JHEP 04 (2010) 076 [arXiv:0906.0003] [INSPIRE].

[41] R. Blumenhagen, T.W. Grimm, B. Jurke and T. Weigand, F-theory uplifts and GUTs, JHEP 09 (2009) 053 [arXiv: 0906.0013] [INSPIRE].

[42] L. Martucci, D-branes on general $N=1$ backgrounds: superpotentials and D-terms, JHEP 06 (2006) 033 [hep-th/0602129] [INSPIRE].

[43] M. Bianchi, A. Collinucci and L. Martucci, Magnetized E3-brane instantons in F-theory, JHEP 12 (2011) 045 [arXiv: 1107.3732] [INSPIRE].

[44] D. Lüst, P. Mayr, S. Reffert and S. Stieberger, F-theory flux, destabilization of orientifolds and soft terms on D7-branes, Nucl. Phys. B 732 (2006) 243 [hep-th/0501139] [INSPIRE].

[45] A.P. Braun, A. Hebecker, C. Lüdeling and R. Valandro, Fixing D7 Brane Positions by F-theory Fluxes, Nucl. Phys. B 815 (2009) 256 [arXiv:0811.2416] [InSPIRE].

[46] A.P. Braun, A. Collinucci and R. Valandro, G-flux in F-theory and algebraic cycles, Nucl. Phys. B 856 (2012) 129 [arXiv:1107.5337] [InSPIRE].

[47] R. Minasian and G.W. Moore, K theory and Ramond-Ramond charge, JHEP 11 (1997) 002 [hep-th/9710230] [INSPIRE].

[48] D.S. Freed and E. Witten, Anomalies in string theory with D-branes, Asian J. Math 3 (1999) 819 [hep-th/9907189] [INSPIRE].

[49] A. Giryavets, S. Kachru, P.K. Tripathy and S.P. Trivedi, Flux compactifications on Calabi-Yau threefolds, JHEP 04 (2004) 003 [hep-th/0312104] [INSPIRE].

[50] M. Kreuzer and H. Skarke, Complete classification of reflexive polyhedra in four-dimensions, Adv. Theor. Math. Phys. 4 (2002) 1209 [hep-th/0002240] [INSPIRE].

[51] S. Gukov, C. Vafa and E. Witten, CFT's from Calabi-Yau four folds, Nucl. Phys. B 584 (2000) 69 [Erratum ibid. B 608 (2001) 477-478] [hep-th/9906070] [INSPIRE].

[52] E. Witten, Phases of $N=2$ theories in two-dimensions, Nucl. Phys. B 403 (1993) 159 [hep-th/9301042] [INSPIRE].

[53] A. Collinucci, F. Denef and M. Esole, D-brane deconstructions in IIB orientifolds, JHEP 02 (2009) 005 [arXiv:0805.1573] [INSPIRE].

[54] M. Kreuzer and H. Skarke, PALP: a package for analyzing lattice polytopes with applications to toric geometry, Comput. Phys. Commun. 157 (2004) 87 [math/0204356] [INSPIRE].

[55] A.P. Braun and N.-O. Walliser, A new offspring of PALP, arXiv:1106.4529 [INSPIRE].

[56] A.P. Braun, J. Knapp, E. Scheidegger, H. Skarke and N.-O. Walliser, PALP - a User Manual, arXiv:1205.4147 [INSPIRE].

[57] J. Tate, Algorithm for determining the type of a singular fiber in an elliptic pencil, in Lecture Notes in Mathematics. Vol. 476: Modular Functions of One Variable IV, Springer, Heidelberg Germany (1975), pg. 33. 
[58] M. Bershadsky et al., Geometric singularities and enhanced gauge symmetries, Nucl. Phys. B 481 (1996) 215 [hep-th/9605200] [INSPIRE].

[59] R. Donagi and M. Wijnholt, Higgs Bundles and UV Completion in F-theory, arXiv:0904.1218 [INSPIRE].

[60] K. Becker, M. Becker, M. Haack and J. Louis, Supersymmetry breaking and alpha-prime corrections to flux induced potentials, JHEP 06 (2002) 060 [hep-th/0204254] [INSPIRE].

[61] S. Krause, C. Mayrhofer and T. Weigand, Gauge Fluxes in F-theory and Type IIB Orientifolds, JHEP 08 (2012) 119 [arXiv:1202.3138] [INSPIRE].

[62] J. Gray et al., Calabi-Yau Manifolds with Large Volume Vacua, arXiv:1207.5801 [INSPIRE].

[63] R. Blumenhagen, S. Moster and E. Plauschinn, Moduli Stabilisation versus Chirality for MSSM like Type IIB Orientifolds, JHEP 01 (2008) 058 [arXiv:0711.3389] [InSPIRE].

[64] R. Blumenhagen, V. Braun, T.W. Grimm and T. Weigand, GUTs in Type IIB Orientifold Compactifications, Nucl. Phys. B 815 (2009) 1 [arXiv:0811.2936] [INSPIRE].

[65] A. Collinucci, M. Kreuzer, C. Mayrhofer and N.-O. Walliser, Four-modulus 'Swiss Cheese' chiral models, JHEP 07 (2009) 074 [arXiv:0811.4599] [INSPIRE].

[66] E. Witten, D-branes and k-theory, JHEP 12 (1998) 019 [hep-th/9810188] [INSPIRE].

[67] G.W. Moore and E. Witten, Selfduality, Ramond-Ramond fields and k-theory, JHEP 05 (2000) 032 [hep-th/9912279] [INSPIRE].

[68] A.M. Uranga, D-brane probes, RR tadpole cancellation and $k$-theory charge, Nucl. Phys. B 598 (2001) 225 [hep-th/0011048] [INSPIRE].

[69] F. Denef, Les Houches Lectures on Constructing String Vacua, arXiv:0803.1194 [INSPIRE].

[70] F. Denef, M.R. Douglas and B. Florea, Building a better racetrack, JHEP 06 (2004) 034 [hep-th/0404257] [INSPIRE].

[71] M. Kreuzer and H. Skarke, Classification of reflexive polyhedra in three-dimensions, Adv. Theor. Math. Phys. 2 (1998) 847 [hep-th/9805190] [inSPIRE].

[72] R. Blumenhagen, B. Jurke, T. Rahn and H. Roschy, Cohomology of Line Bundles: A Computational Algorithm, J. Math. Phys. 51 (2010) 103525 [arXiv:1003.5217] [INSPIRE].

[73] http://wwwth.mppmu.mpg.de/members/blumenha/cohomcalg/, cohomCalg, High-performance line bundle cohomology computation based on [72] (2010).

[74] S. Katz, On the finiteness of rational curves on quintic threefolds, Compos. Math. 60 (1986) 151.

[75] M. Dine, N. Seiberg and E. Witten, Fayet-Iliopoulos Terms in String Theory, Nucl. Phys. B 289 (1987) 589 [INSPIRE].

[76] H. Jockers and J. Louis, D-terms and F-terms from D7-brane fluxes, Nucl. Phys. B 718 (2005) 203 [hep-th/0502059] [INSPIRE].

[77] S.H. Katz and C. Vafa, Geometric engineering of $N=1$ quantum field theories, Nucl. Phys. B 497 (1997) 196 [hep-th/9611090] [INSPIRE].

[78] R. Kallosh, A.-K. Kashani-Poor and A. Tomasiello, Counting fermionic zero modes on M5 with fluxes, JHEP 06 (2005) 069 [hep-th/0503138] [INSPIRE]. 
[79] T.W. Grimm and J. Louis, The effective action of $N=1$ Calabi-Yau orientifolds, Nucl. Phys. B 699 (2004) 387 [hep-th/0403067] [INSPIRE].

[80] B.R. Greene and M. Plesser, Duality in Calabi-Yau moduli space, Nucl. Phys. B 338 (1990) 15 [INSPIRE].

[81] P. Candelas, A. Font, S.H. Katz and D.R. Morrison, Mirror symmetry for two parameter models. 2., Nucl. Phys. B 429 (1994) 626 [hep-th/9403187] [InSPIRE].

[82] D. Martinez Pedrera, D. Mehta, M. Rummel and A. Westphal, to appear.

[83] D. Mehta, Y.-H. He and J.D. Hauenstein, Numerical Algebraic Geometry: A New Perspective on String and Gauge Theories, JHEP 07 (2012) 018 [arXiv:1203.4235] [INSPIRE].

[84] C. Mayrhofer, Compactifications of Type IIB String Theory and F-Theory Models by Means of Toric Geometry, Ph.D. Thesis, Vienna University of Technology, Vienna Austria (2010).

[85] A. Collinucci and R. Savelli, On flux quantization in F-theory, JHEP 02 (2012) 015 [arXiv: 1011.6388] [INSPIRE].

[86] A. Collinucci and R. Savelli, On flux quantization in f-theory II: unitary and symplectic gauge groups, JHEP 08 (2012) 094 [arXiv: 1203.4542] [INSPIRE].

[87] R. Tatar and W. Walters, GUT theories from Calabi-Yau 4-folds with $\mathrm{SO}(10)$ Singularities, arXiv: 1206.5090 [INSPIRE].

[88] P. Candelas, E. Perevalov and G. Rajesh, Toric geometry and enhanced gauge symmetry of F-theory/heterotic vacua, Nucl. Phys. B 507 (1997) 445 [hep-th/9704097] [InSPIRE].

[89] S. Krause, C. Mayrhofer and T. Weigand, $G_{4}$ flux, chiral matter and singularity resolution in F-theory compactifications, Nucl. Phys. B 858 (2012) 1 [arXiv:1109.3454] [INSPIRE].

[90] P. Candelas and X.C. de la Ossa, Comments on conifolds, Nucl. Phys. B 342 (1990) 246 [INSPIRE]. 